Math. Struct. in Comp. Science (2008), vol. 18, pp. 1-34. (C) 2008 Cambridge University Press doi:10.1017/S0960129508006750 Printed in the United Kingdom

\title{
iRho: an imperative rewriting calculus
}

\author{
LUIGI LIQUORI and BERNARD PAUL SERPETTE
}

INRIA Sophia Antipolis, 2004 Route des Lucioles, FR-06902 France

Email: \{Luigi.Liquori;Bernard.Serpette\}@inria.fr

Received 26 January 2006; revised 27 April 2007

\begin{abstract}
We propose an imperative version of the Rewriting Calculus, a calculus based on pattern matching, pattern abstraction and side effects, which we call iRho.

We formulate both a static and big-step call-by-value operational semantics of iRho. The operational semantics is deterministic, and immediately suggests how an interpreter for the calculus may be built. The static semantics is given using a first-order type system based on a form of product types, which can be assigned to term-like structures (that is, records).

The calculus is $\grave{a}$ la Church, that is, pattern abstractions are decorated with the types of the free variables of the pattern.

iRho is a good candidate for the core of a pattern-matching imperative language, where a (monomorphic) typed store can be safely manipulated and where fixed points are built into the language itself.

Properties such as determinism of the interpreter and subject-reduction have been completely checked using a machine-assisted approach with the Coq proof assistant. Progress and decidability of type checking are proved using pen and paper.
\end{abstract}

\section{Introduction}

The study of rewriting-based languages (such as Elan (Protheo Team 2005), Maude (Maude Team 2005), ASF+SDF (van Deursen et al. 1996; Asf+Sdf Team 2005) and

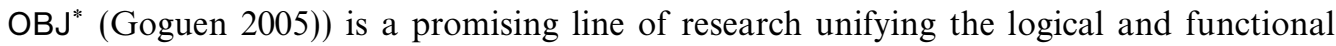
paradigms.

Although rewriting-based languages are less popular than object-oriented languages such as Java (Sun 2005) and C\# (Microsoft 2005) for ordinary programming, they can serve as common typed intermediate languages for implementing compilers for rewritingbased, functional, object-oriented, logic, and other high-level modern languages (Cirstea et al. 2001a; Cirstea et al. 2002; Cirstea et al. 2004).

Pattern matching has been used widely in functional and logic programming (for example, in ML (Milner et al. 1997; Cristal Team 2005), Haskell (Jones 2003), Scheme (R. Kelsey 1998), Curry (Hanus 1997) and Prolog (Kowalski 1979)), but it has generally been considered to be a convenient mechanism for expressing complex requirements about the function's argument, rather than as a basis for an ad hoc paradigm of computation.

One of the main advantages of rewriting-based languages is pattern matching, which allows one to discriminate between alternatives. These languages permit non-determinism in the sense that they can represent a collection of results. That is, pattern matching need not be exclusive, multiple branches can be 'fired' simultaneously. An empty collection of 
results represents an application failure, a singleton represents a deterministic result, and a collection with more than one element represents a non-deterministic choice between the elements of the collection.

This feature highlights a difference compared with those functional languages featuring pattern matching, such as ML, Haskell and Scheme. It shares some similarities with backtracking and exhaustive proof search in logic languages like Prolog. It is possible to make a pair of two functions having the same pattern, and when the pair is applied to an argument, both functions will be fired. Optimistic and pessimistic operational semantics ${ }^{\dagger}$, with a fixed strategy, can then be imposed on the language by defining successful results as Cartesian products that have, in the optimistic case, at least one component that is not a failure value, or, in the pessimistic case, none of its components are failure values. It should then be possible to obtain a logic language on top of this structure by defining an appropriate strategy for backtracking. Useful applications lie in the field of pattern recognition and the manipulation of strings and trees.

The Rewriting Calculus (Rho) (Cirstea et al. 2001b; Cirstea et al. 2004) integrates matching, rewriting and functions in a uniform way; its abstraction mechanism is based on rewrite rule formation - in a term of the form $P \rightarrow A$, one abstracts over all the free variables of the pattern $P$ (instead of over a simple variable as in the lambda calculus). The Rewriting Calculus is a generalisation of the lambda calculus since one may choose the pattern $P$ to be a variable. If an abstraction $P \rightarrow A$ is applied to the term $B$, the evaluation mechanism is based on:

1 bind the free variables present in $P$ to appropriate subterms of $B$ to build a substitution $\theta$; and then

2 apply $\theta$ to $A$.

Indeed, this binding is achieved by matching $P$ against $B$. In rewriting-based languages, pattern matching can be 'customisable' with more sophisticated matching theories, for example, building-in associativity and/or commutativity of equality.

The original Rho calculus is computationally complete, and, through pattern matching, lambda calculus and fixed points can be encoded and type checked using ad hoc patterns. In fact, Rho is a direct generalisation of the core of a typed (rewriting-based and functional) programming language (of the MLUElan family) in which, roughly speaking, an ML-like let becomes by default a letrec by abstracting over a suitable pattern $P$; through pattern matching, one can type check many divergent terms (such as $\Omega$, see Example 6). One of the main features of the Rewriting Calculus is that it can deal with structuring and destructuring structures, such as lists (we record only the names of the constructor and discard the names of the accessors). Since structures are built into the calculus, it follows that the encoding of constructor/accessors is simpler than the standard encoding in the lambda calculus. Table 1 provides an informal comparison between the untyped encoding of accessors in the two formalisms.

\footnotetext{
$\dagger$ 'Optimistic' means that, if a matching failure occurs, the computation is not halted; this is in contrast with a 'pessimistic' machine, that 'kills' the computation once a failure value is produced.
} 


$$
\begin{array}{clr}
\text { ops/form } & \text { Rho calculus } & \text { Lambda calculus } \\
\text { cons } & X \rightarrow Y \rightarrow(\text { cons } X Y) & \lambda X . \lambda Y . \lambda Z . Z X Y \\
\text { car } & \text { (cons } X Y) \rightarrow X & \lambda Z . Z(\lambda X . \lambda Y . X) \\
\text { cdr } & \text { (cons } X Y) \rightarrow Y & \lambda Z . Z(\lambda X . \lambda Y . Y)
\end{array}
$$

Table 1. Accessors and destructors in Rho/lambda calculi

\section{Original contribution}

In this paper we present the first version of the Imperative Rewriting-calculus (iRho), an extension of Rho with references, memory allocation and assignment à la ML (Felleisen and Friedman 1989). To our knowledge, no similar study exists in the literature. The iRho-calculus is a powerful calculus at both the syntactic and semantic levels. It includes all the features of functional/rewriting-based languages with imperative aspects and pattern-matching facilities.

The controlled use of references, in the style of the ML language (Milner et al. 1997) also gives the user the programming ease and expressiveness that, a priori, might not be expected from such a simple calculus.

The crucial ingredients of iRho are a combination of:

(i) modern and safe imperative features, which give full control over the internal datastructure representation; and

(ii) 'matching power', which provides the main Lisp-like operations, such as cons/car/cdr.

The language iRho provides a good theoretical foundation for an emerging family of languages that combine rewriting, functions and patterns with semi-structured XMLdata (for example, XDUCE (Xduce Team 2005), CDUCE (Frisch 2005)) or combining object-orientation and patterns with semi-structured data (for example, HYDROJ (Lee et al. 2003) $)^{\dagger}$.

\section{From theory to practice and vice versa}

We present both a static and dynamic semantics of iRho. The dynamic semantics is given using a natural deduction system (big-step) (Martin-Löf 1984; Milner 1986-87; Plotkin 1981; Tofte 1987; Kahn 1987; Plotkin 2004). The formalisation uses environments inside 'closure values' to keep the value of free variables in function bodies, and a global store to model the imperative traits. In this design phase we have tried not to forget the needs and objectives of a future implementor of the language: that is, to build a sound machine (the interpreter) with a sound type system (the type checker), respecting Milner's slogan that 'well-typed programs do not go wrong', but also taking a bit of care over performance issues.

The static and dynamic semantics are suitable for a mathematical specification, for implementation with high-level programming languages, such as Bigloo (Serrano 2005)

\footnotetext{
$\dagger$ ‘...object-oriented pattern matching naturally focuses on the essential information in a message and is insensitive to inessential information...'
} 
(of the Scheme family), and for certification with a modern and semi-automatic proof assistant, such as Coq (Logical Team 2005).

With this goal in mind, we have encoded the static and dynamic semantics of iRho in Coq. All subtle aspects, which are usually 'swept under the carpet' when working on paper, are here highlighted by the rigid discipline imposed by the Logical Framework of Coq. This process has often influenced the design of the semantics. The continuous interplay of mathematics and manual (that is, pen and paper) $v$ s. mechanical proofs, and prototype implementations using high-level languages such as Scheme (and back) has been fruitful from the very beginning of our project. Although our calculus is rather simple, we expect in the near future to scale our work up to larger projects, such as the certified implementation of compilers for a programming language of the $\mathrm{C}$ family (Cristal Team 2003; Leroy 2005).

Therefore, the main contributions of this paper are as follows:

- We provide a typed framework that enhances the functional language Rho with imperative features such as referencing, dereferencing and assignment operators.

- We enrich the type system with dereferencing and product types. The resulting calculus iRho is a good candidate for giving a semantics to a broad family of functional, rewriting and logic-based languages.

\section{Road map}

The paper is structured as follows. In Sections 2 and 3, we present the syntax and operational semantics of the functional rewriting calculus Rho and the imperative rewriting calculus iRho, respectively. Section 4 describes the type system. Section 5 presents the metatheory for iRho. Section 6 contains various examples of terms, reductions and type checking. Section 7 presents the formalisation of iRho in Coq. Section 8 contains some remarks about our methodology and describes some 'views' of the natural semantics, presents conclusions and lays out some ideas for further work.

The Coq encoding of the dynamic and static semantics (with their theorems) and the prototype implementation of an interpreter in Bigloo can be found at http://www-sop. inria.fr/mascotte/Luigi.Liquori/iRho.

\section{The functional Rho}

For pedagogical reasons we will begin by presenting the functional Rho. This will allow us to introduce almost all the ingredients and technicalities needed to scale-up to the full imperative iRho. In a nutshell, Rho is a functional calculus with pattern matching, and can be seen as the kernel of any (statically typed) programming language based on functions, term rewriting, and pattern matching; many term rewriting systems can also be encoded in iRho (see Cirstea et al. (2004) for the exact class of term rewriting systems that can be encoded). Since the presentation of Rho mimics our current implementation, we make use of closures, that is, <pattern abstraction - environment $>$ pairs, to denote functional values of free variables recorded in the environment. This representation will avoid the use of meta-substitution everywhere. 


$$
\begin{array}{ll}
\tau::=\mathrm{b}|\tau \rightarrow \tau| \tau \wedge \tau & \text { Types } \\
\Delta::=\emptyset|\Delta, X: \tau| \Delta, a: \tau & \text { Contexts } \\
P::=X|a \bar{P}| P, P & \text { Patterns } \\
A::=a|X| P \rightarrow \Delta A|A A| A, A & \text { Terms }
\end{array}
$$

Fig. 1. Rho's syntax.

\subsection{Functional syntax}

Notational conventions. We use the meta-symbols $\rightarrow$ (function and type abstraction), and ',' (structure operator), and the implicit @ (application operator). We assume that the application operator @ associates to the left, while the other operators associate to the right. The priority of @ is higher than that of $\rightarrow$, which is, in turn, of higher priority than ','.

The symbols:

- $A, B, C, \ldots$ range over the set $\mathscr{T}_{A}$ of terms;

$-X, Y, Z, \ldots$, SELF,$\ldots$ range over the set $\mathscr{X}$ of variables $\left(\mathscr{X} \subseteq \mathscr{T}_{A}\right)$;

- $a, b, c, \ldots$, cons, true, false, not, and, or, dummy, ... range over a set $\mathscr{K}$ of term constants $\left(\mathscr{K} \subseteq \mathscr{T}_{A}\right)$;

- $P$ ranges over the set $\mathscr{P}$ of pseudo-patterns, $(\mathscr{X} \subseteq \mathscr{P})$;

- $\tau$ range over the set $\mathscr{T}_{\tau}$ of types, the symbol $\mathrm{b}$ ranges over the set of type constants, the symbols $\Gamma, \Delta$ ranges over contexts;

- $A_{\mathrm{v}}, B_{\mathrm{v}}, C_{\mathrm{v}}, \ldots$ range over the set $\mathscr{V}$ al of values.

We sometimes write $\bar{A}$ for $A_{1} \cdots A_{n}$, for $n \geqslant 0$. The symbol $\equiv$ denotes syntactic equality. The syntax of Rho is presented in Figure 1.

Types and contexts. The symbol $\mathrm{b}$ is used to denote basic types, the arrow type $\tau_{1} \rightarrow \tau_{2}$ is the type of pattern abstractions $P \rightarrow_{\Delta} A$, with $\Delta$ containing the types of the free variables of $P$, and the product type $\tau_{1} \wedge \tau_{2}$ is the type of structure terms $\left(A_{1}, A_{2}\right)$.

Patterns. An unrestricted use of patterns in lambda abstraction may lead to a failure of confluence in small-step semantics (see Klop (1980)). To retain confluence, Vincent van Oostrom (van Oostrom 1990) introduced a suitable syntactical condition on the formation of patterns, called the Rigid Pattern Condition (RPC), which

(i) forces patterns to be 'linear' (that is, no double occurrences of free variables, thus avoiding, the pattern $(a X X))$;

(ii) forbids 'active' variables (thus avoiding the pattern $(X P)$ ).

Not all functional languages with pattern matching apply the same restrictions concerning linearity in patterns. For example, Scheme accepts non-linear patterns, permitting the comparison of subparts of the datum (through eq?), while ML enforces linearity in patterns (but when guards can be used to test for equality between two parts of a data structure). Haskell, because of its lazy evaluation strategy, only accepts patterns 
that are linear. The solution we adopt in this formalisation and implementation of the Rewriting Calculus was influenced by the choice of the implementation language of our operational semantics, namely Scheme. The specification of the matching algorithm in iRho accepts non-linear patterns, and compares subparts of the datum (through $\equiv$, which is implemented using the primitive equiv? in Scheme). Confluence is preserved thanks to the call-by-value strategy of the operational semantics.

The shape of patterns has been limited to algebraic terms and structures (that is, no function as pattern). This restriction is strictly related to the current software development of our interpreter, and to the current mechanical development of the metatheory underlying iRho, and not to theoretical problems (see Barthe et al. (2003)).

Terms. The main intuitions behind the term syntax are as follows:

- Variable and Constant are exactly as in the lambda calculus with constants.

- Structure allows one to express lists, sets, objects, and so on.

- Pattern Abstraction allows one to match over patterns. This gives a conservative extension of the simply-typed lambda calculus when the pattern is a simple variable, that is, $\lambda X: \tau . A \simeq X \rightarrow X: \tau A$; the context $\Delta$ in the pattern abstraction records the types of all the free variables of $P$ (possibly bound in the body $A$ ). For example, the accessors car (in a homogeneous list) can be written in Rho as follows:

$$
\operatorname{car} \triangleq(\text { cons } \mathrm{XY}) \rightarrow \rightarrow_{\Delta} \mathrm{X} \text { with } \Delta \equiv \mathrm{X}: \tau, \mathrm{Y}: \tau^{\prime} .
$$

- Application allows one to apply a pattern abstraction $P \rightarrow_{\Delta} A$ to an argument $B$, which of course must match on $P$. The terms are reduced under a classical call-byvalue evaluation strategy; in the evaluation, the body of a pattern abstraction is not evaluated until the function is called on a suitable value (that is, pattern abstractions are values). For example, $(\operatorname{car}(\operatorname{car}(\operatorname{cons}($ cons a b) c))) will reduce to a.

Observe that compared with 'non-strategic' implementations of the Rewriting Calculus (Cirstea et al. 2001a; Cirstea et al. 2001b; Cirstea et al. 2002; Barthe et al. 2003), the delayed matching constraint $\left[P \ll_{\Delta} A\right] . B$, now just becomes syntactic sugar for $\left(P \rightarrow{ }_{\Delta} A\right) B$ (which is omitted from the source language but will still be present in the set of output values).

Values and environments. The set $\mathscr{V}$ al of values, and the set of environments $\mathscr{E} n$ are defined below, where the last two forms are closures:

$$
A_{\vee}::=a \bar{A}_{\mathrm{\vee}}\left|A_{\mathrm{\vee}}, A_{\mathrm{\vee}}\right|\left\langle P \rightarrow_{\Delta} A . \rho\right\rangle \mid\left\langle\left[P \ll_{\Delta} A_{\mathrm{\vee}}\right] . B \cdot \rho\right\rangle \quad \text { functional values. }
$$

Environments (denoted by $\rho$ ) are partial functions from the set of variables to the set of values, that is, $\rho \in \mathscr{E} n v \simeq[\mathscr{X} \Rightarrow \mathscr{V} a l]_{\perp}$. The extension of an environment is denoted by $\rho\left[X \mapsto A_{\vee}\right]$ and is defined by

$$
\rho\left[X \mapsto A_{\vee}\right](Y) \triangleq \begin{cases}A_{\vee} & \text { if } X \equiv Y \\ \rho(Y) & \text { otherwise }\end{cases}
$$




\subsection{The Rosetta Stone}

The Rewriting Calculus is known to be a conservative extension of the lambda calculus (Cirstea et al. 2001a; Cirstea and Kirchner 2001). Nevertheless, it is typically presented using an infix notation, using as binder the meta-symbol 'arrow' $(\rightarrow)$, instead of the prefix notation using as a binder the meta-symbol 'lambda' $(\lambda)$ together with the meta-symbol 'point' (.). Moreover, since an abstraction can bind more than one variable, the type decoration of a pattern is given by a 'context' $(\Delta)$ instead of a simple type. The rationale is

$$
\begin{aligned}
\lambda X: \underbrace{X: \tau_{1}}_{\Delta} \cdot A & \simeq X \rightarrow_{\Delta} A \quad \text { variables as patterns } \\
\lambda(f X Y): \underbrace{X: \tau_{1}, Y: \tau_{2}}_{\Delta} . A & \simeq(f X Y) \rightarrow \Delta A \text { algebraic patterns. }
\end{aligned}
$$

Since the context $\Delta$ declares the types of all the free variables of $P$, we have

$$
\mathrm{Fv}\left(P \rightarrow_{\Delta} A\right) \triangleq \mathrm{Fv}(A) \backslash \mathrm{Fv}(P) .
$$

The other cases of the Fv definition are given in Definition 1.

Let-like and conditional constructs. Let-like constructs can be generalised to include patterns by viewing them as syntactic sugar for applications, that is,

$$
\text { let } P \ll A \text { in } B \triangleq(P \rightarrow B) A \text {. }
$$

Conditionals can also be easily encoded in Rho, using pairing and application (true, and false are constants), that is,

$$
\text { neg } \triangleq \text { (true } \rightarrow \text { false } \text {, false } \rightarrow \text { true })
$$

if $A$ then $B$ else $C \triangleq($ true $\rightarrow((X \rightarrow B)$ dummy $)$, false $\rightarrow((X \rightarrow C)$ dummy $)) A$.

Observe that, because of the call-by-value strategy, the then and else branches are wrapped in a dummy abstraction, and $X$ is fresh in $B$ and $C$, that is, $X \notin \operatorname{Fv}(B) \cup \operatorname{Fv}(C)$.

Pair encoding. It is also well known that structures can be easily encoded in the lambda calculus using the standard pair encoding.

The 'Rosetta' stone (see Figure 2) gives an intuitive comparison between the lambda-like and rewriting-like notations, with a particular focus on the pair/projection encoding.

\subsection{Functional operational semantics}

We define a big-step call-by-value operational semantics through a natural proof deduction system. The purpose of the deduction system is to map every expression into a value. The semantics is defined using three judgments with the shape

$$
\rho \vdash A \Downarrow_{\text {val }} A_{\vee} \text { or } \quad \vdash\left\langle A_{\mathrm{v}} \cdot B_{\mathrm{v}}\right\rangle \Downarrow_{\text {call }} C_{\mathrm{v}} \text { or } \rho \vdash\left\langle A \cdot A_{\mathrm{v}}\right\rangle \Downarrow_{\text {match }} \rho^{\prime} .
$$

The first judgment evaluates a term in Rho, the second applies one value to another, producing a result value, and the last updates a correct environment obtained by matching 
Context Syntax

$\Delta \quad:=\emptyset|\Delta, X: A| \Delta, a: A$

Rewriting-like Syntax

$A \quad:=P \rightarrow \Delta A|\quad A, B \quad| \overbrace{\left(\left(X_{1}, X_{2}\right) \rightarrow \Delta X_{1,2}\right)(A, B)}^{\text {proj }} \mid \ldots$

Lambda-like Syntax

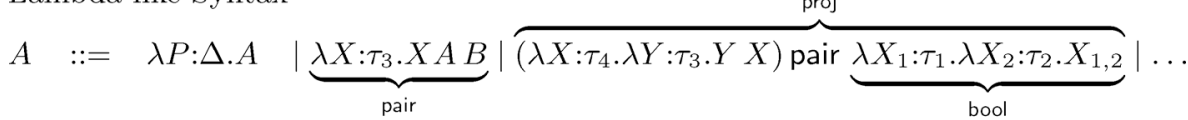

with $\Delta \equiv X_{1}: \tau_{1}, X_{1}: \tau_{2}$ and $\tau_{3} \equiv \tau_{1} \rightarrow \tau_{2} \rightarrow \tau_{1,2}$ and $\tau_{4} \equiv \tau_{3} \rightarrow \tau_{1,2}$

Fig. 2. The Rosetta (functional) Stone.

a term against a value. All the rules are presented in Figure 3, where the symbol $\star$ stands for either @ or ', . In a nutshell:

- (Red.Val) evaluates every constant to itself.

- (RedVVar) simply fetches the value of $X$ into the environment.

- (Red.Struct) simply evaluates a structure to a structure value.

- (Red.Fun) evaluates a pattern abstraction to a closure.

- (Red.Appl $l_{\mathrm{v}}$ ) first reduces the term $A$ to a value $A_{\mathrm{v}}$, then evaluates the argument $B$ in $B_{\mathrm{v}}$, and finally applies $A_{\mathrm{v}}$ to $B_{\mathrm{v}}$ using the $\Downarrow_{\text {call }}$ judgment.

- (Call-Algbr) builds an algebraic value under the shape of an application in weak head-normal form.

- (Call-Struct) applies every element of the structure value to the argument $C_{\mathrm{v}}$.

- (Call-FunOk) first matches successfully $P$ against $B_{\mathrm{v}}$, and then evaluates the body of the pattern abstraction $A$ in the new environment calculated by $\Downarrow_{\text {match }}$.

- (Call-FunKo) applies when the match of $P$ against $B_{\vee}$ fails: a failure value is returned.

- (Call-Wrong) applies a failure value to a value; the failure value is then propagated.

- (Match.Const) means that matching two equal constants does not modify the resulting environment.

- (Match·VarNew) and (Match.VarEq) mean that matching a variable against a value produces an environment updated with the new binding, or the same environment if the variable is already bound with exactly the same value.

- (Match-Pair) means that matching either an application or a structure (recall that $\star \in$ $\{$ @ , \}) produces an environment resulting from the composition of two environments.

The natural operational semantics is deterministic and immediately suggests how to build an interpreter. The standard first-order matching algorithm of Huet (1976) is implemented by a judgment $\rho \vdash\left\langle A . A_{\mathrm{v}}\right\rangle \Downarrow_{\text {match }} \rho^{\prime}$ that, given an environment, a term and a value, either enriches the environment or fails. However, the algorithm has been enhanced in order to take into account the imperative features of the calculus.

Remark 1 (On failure values and exceptions). 'Failure values' $\left\langle\left[P \ll_{\Delta} A_{\mathrm{v}}\right] . B . \rho\right\rangle$ denote failures occurring when we cannot find a correct substitution $\theta$ on the free variables of $P$ such that $\theta(P) \equiv A_{\mathrm{v}}$; the environment $\rho$ records the value of the free variables of $B$. 
Value reduction $\Downarrow_{\text {val }}$

$$
\begin{aligned}
& \left.\frac{X \in \operatorname{Dom}(\rho)}{\rho \vdash a \Downarrow_{\text {val }} a}(\operatorname{Red} \cdot \text { Val }) \quad \text { Red.Var }\right) \\
& \frac{\rho \vdash A \Downarrow_{\text {val }} A_{\mathrm{v}} \quad \rho \vdash B \Downarrow_{\text {val }} B_{\mathrm{v}}}{\rho \vdash A, B \Downarrow_{\text {val }} A_{\mathrm{v}}, B_{\mathrm{v}}} \text { (Red.Struct) } \\
& \overline{\rho \vdash P \rightarrow \Delta A \Downarrow_{\text {val }}\langle P \rightarrow \Delta A \cdot \rho\rangle} \text { (Red.Fun) } \\
& \frac{\rho \vdash A \Downarrow_{\text {val }} A_{\mathrm{v}} \quad \rho \vdash B \Downarrow_{\text {val }} B_{\mathrm{v}} \vdash\left\langle A_{\mathrm{v}}, B_{\mathrm{v}}\right\rangle \Downarrow_{\text {call }} C_{\mathrm{v}}}{\rho \vdash A B \Downarrow_{\text {val }} C_{\mathrm{v}}}\left(\operatorname{Red} \cdot \mathrm{Appl}_{\mathrm{v}}\right)
\end{aligned}
$$

Call reduction $\Downarrow_{\text {call }}$

$$
\begin{aligned}
& \overline{\vdash\left\langle a \bar{A}_{\mathrm{v}} \cdot B_{\mathrm{v}}\right\rangle \Downarrow_{\text {call }} a \bar{A}_{\mathrm{v}} B_{\mathrm{v}}} \text { (Call.Algbr) } \\
& \frac{\vdash\left\langle A_{\mathrm{v}} \cdot C_{\mathrm{v}}\right\rangle \Downarrow_{\text {call }} D_{\mathrm{v}} \quad \vdash\left\langle B_{\mathrm{v}} \cdot C_{\mathrm{v}}\right\rangle \Downarrow_{\text {call }} E_{\mathrm{v}}}{\vdash\left\langle\left(A_{\mathrm{v}}, B_{\mathrm{v}}\right) \cdot C_{\mathrm{v}}\right\rangle \Downarrow_{\text {call }} D_{\mathrm{v}}, E_{\mathrm{v}}} \text { (Call-Struct) } \\
& \frac{\rho \vdash\left\langle P \cdot B_{\mathrm{v}}\right\rangle \Downarrow_{\text {match }} \rho^{\prime} \quad \rho^{\prime} \vdash A \Downarrow_{\text {val }} A_{\mathrm{v}}}{\vdash\left\langle\langle P \rightarrow \Delta A \cdot \rho\rangle \cdot B_{\mathrm{v}}\right\rangle \Downarrow_{\text {call }} A_{\mathrm{v}}} \text { (Call.FunOk) } \\
& \frac{\nexists \rho^{\prime} \cdot \rho \vdash\left\langle P \cdot B_{\mathrm{v}}\right\rangle \Downarrow_{\text {match }} \rho^{\prime}}{\vdash\left\langle\langle P \rightarrow \Delta A \cdot \rho\rangle \cdot B_{\mathrm{v}}\right\rangle \Downarrow_{\text {call }}\left\langle\left[P \ll \Delta B_{\mathrm{v}}\right] \cdot A \cdot \rho\right\rangle} \text { (Call-FunKo) } \\
& \overline{\vdash\left\langle\left\langle\left[P \ll \Delta B_{\mathrm{v}}\right] \cdot A \cdot \rho\right\rangle \cdot C_{\mathrm{v}}\right\rangle \Downarrow_{\text {call }}\left\langle\left[P \ll \Delta B_{\mathrm{v}}\right] \cdot A \cdot \rho\right\rangle} \text { (Call·Wrong) }
\end{aligned}
$$

Matching reduction $\Downarrow_{\text {match }}$

$$
\begin{gathered}
\frac{\rho \vdash\langle a \cdot a\rangle \Downarrow_{\text {match }} \rho}{\rho \vdash} \text { (Match.Const) } \\
\frac{\rho(X)=\perp}{\rho \vdash\left\langle X \cdot A_{\mathrm{v}}\right\rangle \Downarrow_{\text {match }} \rho\left[X \mapsto A_{\mathrm{v}}\right]} \text { (Match.VarNew) } \\
\frac{\rho_{0} \vdash\left\langle A \cdot A_{\mathrm{v}}\right\rangle \Downarrow_{\text {match }} \rho_{1} \quad \rho_{1} \vdash\left\langle B \cdot B_{\mathrm{v}}\right\rangle \Downarrow_{\text {match }} \rho_{2}}{\rho \vdash\left\langle X \cdot A_{\mathrm{v}}\right\rangle \Downarrow_{\text {match }} \rho} \text { (Match.VarEq) } \\
\rho_{0} \vdash\left\langle A \star B \cdot A_{\mathrm{v}} \star B_{\mathrm{v}}\right\rangle \Downarrow_{\text {match }} \rho_{2}
\end{gathered}
$$

Fig. 3. Natural functional semantics. 
Failure values are obtained during the computation when a matching failure occurs. They can in principle be discarded, or caught by an exception handler (see Cirstea et al. (2002)), which can be implemented in the interpreter.

In this paper, for the sake of simplicity, we will not deal with pattern-mismatch errors and pattern exceptions (but this feature is available as an option in our interpreter). In the examples of Section 6, when a computation terminates with success (that is, not a failure value), all intermediate failure values are simply discharged from the final output. The interested reader may consult Cirstea et al. (2004) for the necessary operational semantics extensions/enhancements, and for a suitable matching theory that automatically drops failure values.

Remark 2 (Optimistic vs. pessimistic vs. clean machines). Our natural semantics is 'optimistic', in the sense that if a matching failure occurs, the computation is not halted and we explicitly list such a failure in the final result. Of course, other choices are possible, such as 'killing' the computation once a failure value is produced, or 'cleaning' all failure values from the final result. The distinction is clearly visible when dealing with structures, as shown in the following trivial example (we let $\Downarrow_{\text {val }}^{\text {opt }}$ and $\Downarrow_{\text {val }}^{\text {pex }}$ and $\Downarrow_{\text {val }}^{\text {clean }}$, denote the optimistic, pessimistic and clean machine cases, respectively)

$$
\begin{gathered}
\frac{\vdots}{\varnothing \vdash(3 \rightarrow 3,4 \rightarrow 4) 4 \Downarrow_{\text {val }}^{\text {opt }}\langle[3 \ll 4] .3 . \varnothing\rangle, 4} \\
\frac{\vdots}{\varnothing \vdash(3 \rightarrow 3,4 \rightarrow 4) 4 \Downarrow_{\text {val }}^{\text {pex }}\langle[3 \ll 4] .3 . \varnothing\rangle} \\
\frac{\vdots}{\varnothing \vdash(3 \rightarrow 3,4 \rightarrow 4) 4 \Downarrow_{\text {val }}^{\text {clean }} 4}
\end{gathered}
$$

We conclude this section with a simple example of two functional evaluations.

Example 1 (Two functional evaluations). Consider the following term in Rho

$$
((a X) \rightarrow(3 \rightarrow 3) X)(a 3) \quad \text { and } \quad((a X) \rightarrow(3 \rightarrow 3) X)(a 4)
$$

and let

$$
\begin{aligned}
\odot & \equiv \varnothing \vdash\langle((a X) \rightarrow(3 \rightarrow 3) X) . \varnothing\rangle \Downarrow_{\text {val }}\langle((a X) \rightarrow(3 \rightarrow 3) X) . \varnothing\rangle \\
\triangle & \equiv \varnothing \vdash(a 3) \Downarrow_{\text {val }}(a 3) \\
\square & \equiv \varnothing \vdash(a 4) \Downarrow_{\text {val }}(a 4) \\
A_{\vee} & \equiv\langle[3 \ll 4] .3 . \rho\rangle \quad \rho_{0} \triangleq[X \mapsto 3] \quad \rho_{1} \triangleq[X \mapsto 4] .
\end{aligned}
$$

The deduction trees are shown in Figure 4. 


$$
\begin{aligned}
& \emptyset \vdash\langle a \cdot a\rangle \Downarrow_{\text {match }} \emptyset \\
& \emptyset \vdash\langle X .3\rangle \Downarrow_{\text {match }} \rho_{0} \\
& \rho_{0} \vdash X \Downarrow_{\text {val }} 3 \\
& \emptyset \vdash\langle(a X) \cdot(a 3)\rangle \Downarrow_{\text {match }} \rho_{0} \\
& \rho_{0} \vdash(3 \rightarrow 3) \Downarrow_{\text {val }}\left\langle(3 \rightarrow 3) \cdot \rho_{0}\right\rangle \\
& \rho_{0} \vdash(3 \rightarrow 3) X \Downarrow_{\text {val }} 3 \\
& \vdash\langle\langle((a X) \rightarrow(3 \rightarrow 3) X) \cdot \emptyset\rangle \cdot(a 3)\rangle \Downarrow_{\text {call }} 3 \\
& \emptyset \vdash((a X) \rightarrow(3 \rightarrow 3) X)(a 3) \downarrow_{\text {val }} 3
\end{aligned}
$$$$
\frac{\rho_{0} \vdash\langle 3.3\rangle \Downarrow_{\text {match }} \rho_{0} \quad \rho_{0} \vdash 3 \Downarrow_{\text {val }} 3}{\vdash\left\langle\left\langle(3 \rightarrow 3) \cdot \rho_{0}\right\rangle \cdot 3\right\rangle \Downarrow_{\text {call }} 3}
$$

and

$$
\begin{aligned}
& \frac{\nexists \rho_{2} . \rho_{1} \vdash\langle 3 \cdot 4\rangle \Downarrow_{\text {match }} \rho_{2}}{\vdash\langle\langle(3 \rightarrow 3) \cdot \rho\rangle \cdot 4\rangle \Downarrow_{\text {call }} A_{\vee}} \\
& \emptyset \vdash\langle a \cdot a\rangle \Downarrow_{\text {match }} \emptyset \quad \rho_{1} \vdash X \Downarrow_{\text {val }} 4 \\
& \emptyset \vdash\langle X \cdot 4\rangle \Downarrow_{\text {match }} \rho_{1} \quad \rho_{1} \vdash(3 \rightarrow 3) \Downarrow_{\text {val }}\left\langle(3 \rightarrow 3) \cdot \rho_{1}\right\rangle \\
& \emptyset \vdash\langle(a X) \cdot(a 4)\rangle \Downarrow_{\text {match }} \rho_{1} \quad \rho_{1} \vdash(3 \rightarrow 3) X \Downarrow_{\text {val }} A_{v} \\
& \odot \quad \square \quad \vdash\langle\langle((a X) \rightarrow(3 \rightarrow 3) X) \cdot \emptyset\rangle \cdot(a 4)\rangle \Downarrow_{\text {call }} A_{\vee} \\
& \emptyset \vdash((a X) \rightarrow(3 \rightarrow 3) X)(a 4) \Downarrow_{\text {val }} A_{\vee}
\end{aligned}
$$

Fig. 4. Natural deduction of $((a X) \rightarrow(3 \rightarrow 3) X)(a 3)$ and $((a X) \rightarrow(3 \rightarrow 3) X)(a 4)$.

$$
\begin{array}{lll}
\tau::=\ldots \text { as in Rho... } \mid \tau \text { ref } & \text { Types } \\
\Delta::=\ldots \text { as in Rho... } & \text { Contexts } \\
P::=\ldots \text { as in Rho... } \operatorname{ref} P & \text { Patterns } \\
A::=\ldots \text { as in Rho... } \operatorname{ref} A \mid A:=A & \text { Terms }
\end{array}
$$

Fig. 5. iRho's syntax.

\section{The Imperative Rewriting Calculus}

In this section we add imperative features to our rewriting calculus to yield the full iRho. We extend the syntax of terms by adding (de)referencing and assignment operators, by extending the set of values and contexts including references, by adding new referencetypes, and by recasting our natural semantics in terms of store locations and environments (Tofte 1987; Felleisen and Friedman 1989).

Syntax. The syntax of iRho (types, contexts, patterns and terms) is given in Figure 5. Intuitively, iRho deals with references à la ML that is:

- Ref terms The term ref $A$ is a referencing term (the-location-of); if $A$ is a term of type $\tau$, then $\operatorname{ref} A$ is a pointer to $A$ of type $\tau$ ref; 
- Assignment terms The term $A:=B$ is an assignment operator, which returns as its result the value obtained by evaluating $B$ (as in, for example, SmallTalk); other languages, such as OCaml, return a special value () of type unit.

\subsection{Imperative syntax}

As an immediate benefit of the new and powerful built-in pattern-matching algorithm, the classical dereferencing term (goto-memory), denoted by $! A$, where $A$ is a pointer in the store can be easily defined as follows (types are omitted):

$$
! A \triangleq(\operatorname{ref} X \rightarrow X) A .
$$

This is a nice feature with respect to functional core calculi, in that it mixes imperative and functional features, as in Caml.

Sequencing can be also defined in iRho as follows (types are omitted):

$$
A ; B \triangleq(X \rightarrow B) A \quad X \notin \mathrm{Fv}(B) .
$$

Issues related to garbage collection are beyond the scope of this paper: new locations created during reduction through referencing $(\operatorname{ref} A)$ will remain in the store forever. In principle, the classical techniques of Ian Mason and Carolyn Talcott, and Greg Morrisset et al. (Mason and Talcott 1992; Morrisett et al. 1995) could be applied to iRho.

Values and stores. The set $\mathscr{V}$ al of values is enriched by locations. The symbol $\imath$ ranges over the set $\mathscr{L}_{o c}$ of store locations, and the symbol $\sigma$ ranges over the set of global stores $\mathscr{S}$ tore.

$$
A_{\vee}::=\ldots \text { as in Rho } \ldots \mid l \quad \text { imperative values. }
$$

Stores are partial functions from the set $\mathscr{L}$ of locations to the set of values, that is, $\sigma \in \mathscr{S}$ tore $\simeq[\mathscr{L} o c \Rightarrow \mathscr{V} a l]_{\perp}$; we denote the extension of a store by $\sigma\left[\imath \mapsto A_{\mathrm{v}}\right]$ with the meaning

$$
\sigma\left[\imath \mapsto A_{\vee}\right]\left(l^{\prime}\right) \triangleq \begin{cases}A_{\vee} & \text { if } \iota \equiv \iota^{\prime} \\ \sigma\left(l^{\prime}\right) & \text { otherwise }\end{cases}
$$

\subsection{Imperative operational semantics}

As in the functional case, we define an 'optimistic' big-step operational semantics. Again, the chosen strategy is call by value, and the semantics is defined using three judgments with the shape

$$
\sigma . \rho \vdash A \Downarrow_{\text {val }} A_{\mathrm{v}} \cdot \sigma^{\prime} \quad \text { or } \quad \sigma \vdash\left\langle A_{\mathrm{v}} \cdot B_{\mathrm{v}}\right\rangle \Downarrow_{\text {call }} C_{\mathrm{v}} \cdot \sigma^{\prime} \quad \text { or } \quad \sigma \cdot \rho \vdash\left\langle A \cdot A_{\mathrm{v}}\right\rangle \Downarrow_{\text {match }} \rho^{\prime} .
$$

The main difference compared with the functional calculus Rho is that all judgments have as premise a global store $\sigma$, which can be modified and returned as a result. In the case of $\Downarrow_{\text {val }}$ and $\Downarrow_{\text {call }}$, a store $\sigma$ is given as input, and a (possibly modified) store $\sigma^{\prime}$ is returned as output. In the $\Downarrow_{\text {match }}$ rule, a store $\sigma$ is needed as input since our matching algorithm 
Value reduction $\Downarrow_{\text {val }}$

$$
\begin{aligned}
& \frac{\sigma_{0} \cdot \rho \vdash A \Downarrow_{\text {val }} A_{\mathrm{v}} \cdot \sigma_{1} \quad \sigma_{1} \cdot \rho \vdash B \Downarrow_{\text {val }} B_{\mathrm{v}} \cdot \sigma_{2}}{\sigma_{0} \cdot \rho \vdash A, B \Downarrow_{\text {val }} A_{\mathrm{v}}, B_{\mathrm{v}} \cdot \sigma_{2}} \text { (Red.Struct) } \\
& \frac{\iota \notin \operatorname{Dom}\left(\sigma_{1}\right) \quad \sigma_{0} \cdot \rho \vdash A \Downarrow_{\text {val }} A_{\mathrm{v}} \cdot \sigma_{1}}{\sigma_{0} \cdot \rho \vdash \operatorname{ref} A \Downarrow_{\text {val }} \iota \cdot \sigma_{1}\left[\iota \mapsto A_{\mathrm{v}}\right]}(\operatorname{Red} \cdot \operatorname{Ref}) \\
& \left.\frac{\sigma_{0} \cdot \rho \vdash A \Downarrow_{\text {val }} A_{\mathrm{v}} \cdot \sigma_{1} \quad \sigma_{1} \cdot \rho \vdash B \Downarrow_{\text {val }} B_{\mathrm{v}} \cdot \sigma_{2} \quad \sigma_{2} \vdash\left\langle A_{\mathrm{v}} \cdot B_{\mathrm{v}}\right\rangle \Downarrow_{\text {call }} C_{\mathrm{v}} \cdot \sigma_{3}}{\sigma_{0} \cdot \rho \vdash A B \Downarrow_{\text {val }} C_{\mathrm{v}} \cdot \sigma_{3}} \text { (Red.Appl }\right) \\
& \frac{\sigma_{0} \cdot \rho \vdash A \Downarrow_{\text {val }} \iota \cdot \sigma_{1} \quad \iota \in \operatorname{Dom}\left(\sigma_{1}\right) \quad \sigma_{1} \cdot \rho \vdash B \Downarrow_{\text {val }} B_{\mathrm{v}} \cdot \sigma_{2}}{\sigma_{0} \cdot \rho \vdash A:=B \Downarrow_{\text {val }} B_{\mathrm{v}} \cdot \sigma_{2}\left[\iota \mapsto B_{\mathrm{v}}\right]} \text { (Red.Ass) }
\end{aligned}
$$

Update of (Red·Val), (Red·Fun), (Red·Var) with the unused store parameter.

Call reduction $\Downarrow_{\text {call }}$

$$
\begin{gathered}
\frac{\sigma_{0} \cdot \rho \vdash\left\langle P \cdot B_{\mathrm{v}}\right\rangle \Downarrow_{\text {match }} \rho^{\prime} \quad \sigma_{0} \cdot \rho^{\prime} \vdash A \Downarrow_{\text {val }} A_{\mathrm{v}} \cdot \sigma_{1}}{\sigma_{0} \vdash\left\langle\langle P \rightarrow \Delta A \cdot \rho\rangle \cdot B_{\mathrm{v}}\right\rangle \Downarrow_{\text {call }} A_{\mathrm{v}} \cdot \sigma_{1}} \text { (Call-FunOk) } \\
\frac{\sigma_{0} \vdash\left\langle A_{\mathrm{v}} \cdot C_{\mathrm{v}}\right\rangle \Downarrow_{\text {call }} D_{\mathrm{v}} \cdot \sigma_{1} \quad \sigma_{1} \vdash\left\langle B_{\mathrm{v}} \cdot C_{\mathrm{v}}\right\rangle \Downarrow_{\text {call }} E_{\mathrm{v}} \cdot \sigma_{2}}{\sigma_{0} \vdash\left\langle\left(A_{\mathrm{v}}, B_{\mathrm{v}}\right) \cdot C_{\mathrm{v}}\right\rangle \Downarrow_{\text {call }} D_{\mathrm{v}}, E_{\mathrm{v}} \cdot \sigma_{2}} \text { (Call.Struct) }
\end{gathered}
$$

Update of (Call-FunKo), (Call·Algbr), (Call·Wrong) with the unused store parameter.

Matching reduction $\Downarrow_{\text {match }}$

$$
\frac{\iota \in \operatorname{Dom}(\sigma) \quad \sigma(\iota) \equiv A_{\vee} \quad \sigma \cdot \rho \vdash\left\langle P \cdot A_{\vee}\right\rangle \Downarrow_{\text {match }} \rho^{\prime}}{\sigma \cdot \rho \vdash\langle\operatorname{ref} P \cdot \iota\rangle \Downarrow_{\text {match }} \rho^{\prime}} \text { (Match.Ref) }
$$

Update of (Match·Const), (Match·Var), (Match·Pair) with the unused store parameter.

Fig. 6. Natural imperative semantics.

allows us to match a referencing term ref $A$ to a pointer variable, as in, for example,

$$
\left[l_{0} \mapsto 3\right] .\left[Y \mapsto \imath_{0}\right] \vdash(\operatorname{ref} X \rightarrow X: \mathrm{b} X) Y \Downarrow_{\mathrm{val}} 3 \cdot\left[\imath_{0} \mapsto 3\right]
$$

The rules of the dynamic semantics are defined in Figure 6. In a nutshell:

- $\left(\operatorname{Red} \cdot\left\{v, \mathrm{Appl}_{\mathrm{v}}\right.\right.$, Var, Fun, Struct $\left.\}\right)$ behave, essentially, as in the functional case, except that the store parameter is propagated over the judgments in the premises and in the conclusion. 
- (Red-Ref) first reduces $A$ into a value, and then stores it into a 'fresh' location $t$.

- (Red-Ass) performs assignment: first we reduce the receiver $A$ into an (existing) memory location, then we reduce the expression $B$ (to be assigned) to a value, and, finally, we give as result the value produced by $B$, and a new store, which performs the modification in situ.

- (Call-\{FunOk, Struct, FunKo, Algbr, Wrong $\})$ do not present any surprises when compared with the corresponding rules in Rho: the only difference lies in the store propagation from the input of the conclusion (through the premises) to the output of the conclusion (value $\cdot$ store).

- (Match· $\cdot\{$ Const, VarNew, VarEq, Pair $\}$ ) have the same matching judgments as in Figure 3 with the addition of an (unused) extra parameter $\sigma$.

- (Match.Ref) is the only matching rule that needs a store as an input argument: it first fetches the value $A_{\mathrm{v}}$ in the store $\sigma$, at the location $l$, and then calls the matching of the pattern $P$ against the value $A_{\mathrm{v}}$. An example of imperative pattern matching is

$$
\left[l_{0} \mapsto 3\right] \cdot \varnothing \vdash\left\langle\operatorname{ref} X \cdot l_{0}\right\rangle \Downarrow_{\text {match }}[X \mapsto 3]
$$

This pattern-matching rule allows us to consider the dereferencing term $! A$ simply as sugar in iRho.

Observe that the following rule, which first reduces $A$ into a location value $l$ and then returns the value stored at $l$, is admissible:

$$
\frac{\sigma_{0} \cdot \rho \vdash A \Downarrow_{\text {val }} l \cdot \sigma_{1} \quad l \in \operatorname{Dom}\left(\sigma_{1}\right)}{\sigma_{0} \cdot \rho \vdash ! A \Downarrow_{\text {val }} \sigma_{1}(l) \cdot \sigma_{1}} \text { (Red.Deref). }
$$

We conclude with a simple example of an imperative evaluation.

Example 2 (An imperative evaluation). Take the imperative term

$$
((a(X, Y)) \rightarrow(3 \rightarrow X:=! Y) ! X)(a(\text { ref } 3, \text { ref } 4))
$$

with $\sigma_{0} \triangleq\left[\imath_{0} \mapsto 3\right]\left[l_{1} \mapsto 4\right]$, and $\sigma_{1} \triangleq \sigma_{0}\left[\imath_{0} \mapsto 4\right]$, and $\rho_{0} \triangleq\left[X \mapsto \imath_{0}\right]\left[Y \mapsto \imath_{1}\right]$. The deduction tree is shown in Figure 7.

\section{The type system}

In this section we present a type system for iRho. As usual, we employ type checking to catch some errors before run-time evaluation. In the following section, we present a rich collection of (typable) examples, namely decision procedures, meaningful objects, fixed points and term rewriting systems. The type system can probably be extended with a subtyping relation, or with bounded polymorphism, to capture the behaviour of structuresas-objects, and object-oriented features. Compared with previous type systems for the (functional) Rho (Cirstea et al. 2001b; Cirstea et al. 2002; Barthe et al. 2003; Cirstea et al. 2004), structures can now have different types, that is, thanks to the following typing rule, which is new compared with the previous typed formulations of the Rewriting Calculus:

$$
\frac{\Gamma \vdash_{\mathrm{A}} A: \tau_{1} \quad \Gamma \vdash_{\mathrm{A}} B: \tau_{2}}{\Gamma \vdash_{\mathrm{A}} A, B: \tau_{1} \wedge \tau_{2}} \text { (Term:Struct). }
$$




$$
\begin{aligned}
& \iota_{0} \in \operatorname{Dom}\left(\sigma_{0}\right) \quad \sigma_{0} \cdot \rho_{0} \vdash Y \Downarrow_{\text {val }} \iota_{1} \cdot \sigma_{0} \\
& \sigma_{0} \cdot \rho_{0} \vdash X \Downarrow_{\text {val }} \iota_{0} \cdot \sigma_{0} \quad \sigma_{0} \cdot \rho_{0} \vdash ! Y \Downarrow_{\text {val }} 4 \cdot \sigma_{0} \\
& \sigma_{0} \cdot \rho_{0} \vdash X:=! Y \Downarrow_{\text {val }} 4 \cdot \sigma_{1} \\
& \sigma_{0} \cdot \rho_{0} \vdash\langle 3.3\rangle \Downarrow_{\text {match }} \rho_{0} \\
& \sigma_{0} \vdash\left\langle\left\langle 3 \rightarrow X:=! Y \cdot \rho_{0}\right\rangle \cdot 3\right\rangle \Downarrow_{\text {call }} 4 \cdot \sigma_{1} \\
& \sigma_{0} \cdot \rho_{0} \vdash ! X \Downarrow_{\text {val }} 3 \cdot \sigma_{0} \\
& \sigma_{0} \cdot \rho_{0} \vdash 3 \rightarrow X:=! Y \Downarrow_{\text {val }}\left\langle 3 \rightarrow X:=! Y \cdot \rho_{0}\right\rangle \cdot \sigma_{0} \\
& \sigma_{0}, \rho_{0} \vdash(3 \rightarrow X:=! Y) ! X \Downarrow_{\text {call }} 4 \cdot \sigma_{1} \\
& \sigma_{0} \cdot \emptyset \vdash\left\langle(a(X, Y)) \cdot\left(a\left(\iota_{0}, \iota_{1}\right)\right)\right\rangle \Downarrow_{\text {match }} \rho_{0} \\
& \sigma_{0} \cdot \emptyset \vdash\left\langle\langle((a(X, Y)) \rightarrow(3 \rightarrow X:=! Y) ! X) \cdot \emptyset\rangle \cdot\left(a\left(\iota_{0}, \iota_{1}\right)\right)\right\rangle \Downarrow_{\text {call }} 4 \cdot \sigma_{1} \\
& \emptyset \cdot \emptyset \vdash(a(\operatorname{ref} 3, \text { ref } 4)) \Downarrow_{\text {val }}\left(a\left(\iota_{0}, \iota_{1}\right)\right) \cdot \sigma_{0} \\
& \emptyset . \emptyset \vdash((a(X, Y)) \rightarrow(3 \rightarrow X:=! Y) ! X) \Downarrow_{\text {val }}\langle((a(X, Y)) \rightarrow(3 \rightarrow X:=! Y) ! X) \cdot \emptyset\rangle \cdot \emptyset \\
& \emptyset . \emptyset \vdash((a(X, Y)) \rightarrow(3 \rightarrow X:=! Y) ! X)(a(\operatorname{ref} 3, \operatorname{ref} 4)) \Downarrow_{\text {val }} 4 \cdot \sigma_{1}
\end{aligned}
$$

Fig. 7. Natural deduction of $((a(X, Y)) \rightarrow(3 \rightarrow X:=! Y) ! X)(a($ ref 3 , ref 4$))$.

The type $\tau_{1} \wedge \tau_{2}$, which is reminiscent of a record-types discipline, is suitable for heterogeneous structures, like lists, ordered sets or objects. This enhancement gives a more flexible type discipline, where the product type $\tau_{1} \wedge \tau_{2}$ reflects the implicit noncommutative property of ',' in the term ' $A, B$ ', that is, ' $A, B$ ' does not necessarily behave in the same way as ' $B, A$ '. This modification greatly improves the expressiveness compared with previous typing disciplines on the Rewriting Calculus (Cirstea et al. 2004) in the sense that it gives a type to terms that will not be stuck at run time, but it does complicate the metatheory and mechanical proof development. The main enhancement compared with previous versions of the Rewriting Calculus (Barthe et al. 2003) is that here the elements of the structure are not forced to have the same type.

The type system $\vdash_{\mathrm{A}}$ is algorithmic: the type rules are deterministic and suggest two decision procedures for type reconstruction and type checking. We say that a set of rules specifies a deterministic typing algorithm if the type rules are syntax directed, and each rule satisfies the sub-formula property (all the formulas appearing in the premise of a rule are sub-formulas of those appearing in the conclusion).

The main complication in the type system lies in applying a structure to an argument, thus producing a structure value by dispatching the argument to all the pattern abstractions contained in the structure.

The structure value will be typed with a product type containing all the components of the structure. As a simple example, if we apply a structure (with type $\left(b_{1} \rightarrow b_{2}\right) \wedge\left(b_{1} \rightarrow b_{3}\right)$ ) to an argument of type $b_{1}$, we would obtain as result a structure value of type $b_{2} \wedge b_{3}$. To capture this behaviour (which is a direct consequence of dispatching application into 
Pattern rules

$$
\begin{gathered}
\frac{\Gamma_{1}, \alpha: \tau, \Gamma_{2} \vdash_{\Gamma} o k}{\Gamma_{1}, \alpha: \tau, \Gamma_{2} \vdash_{\mathrm{P}} \alpha: \tau} \text { (Patt.Start) } \frac{\Gamma \vdash_{\mathrm{P}} P_{1}: \tau_{1} \Gamma \vdash_{\mathrm{P}} P_{2}: \tau_{2}}{\Gamma \vdash_{\mathrm{P}} P_{1}, P_{2}: \tau_{1} \wedge \tau_{2}} \text { (Patt.Struct) } \\
\frac{\operatorname{arr}\left(\tau_{1}\right) \equiv \tau_{2} \rightarrow \tau_{3} \quad \Gamma \vdash_{\mathrm{P}} a \bar{P}: \tau_{1} \quad \Gamma \vdash_{\mathrm{P}} P: \tau_{2}}{\Gamma \vdash_{\mathrm{P}} a \bar{P} P: \tau_{3}} \text { (Patt.Algbr) }
\end{gathered}
$$

Term rules

$$
\begin{aligned}
& \frac{\Gamma_{1}, \alpha: \tau, \Gamma_{2} \vdash_{\Gamma} o k}{\Gamma_{1}, \alpha: \tau, \Gamma_{2} \vdash_{\mathrm{A}} \alpha: \tau} \text { (Term·Start) } \quad \frac{\Gamma \vdash_{\mathrm{A}} A: \tau}{\Gamma \vdash_{\mathrm{A}} \operatorname{ref} A: \tau \text { ref }}(\text { Term } \cdot \text { Ref }) \\
& \frac{\Gamma \vdash_{\mathrm{A}} A: \tau \text { ref } \Gamma \vdash_{\mathrm{A}} B: \tau}{\Gamma \vdash_{\mathrm{A}} A:=B: \tau} \text { (Term.Assign) } \frac{\Gamma \vdash_{\mathrm{A}} A: \tau_{1} \Gamma \vdash_{\mathrm{A}} B: \tau_{2}}{\Gamma \vdash_{\mathrm{A}} A, B: \tau_{1} \wedge \tau_{2}} \text { (Term.Struct) } \\
& \operatorname{arr}\left(\tau_{1}\right) \equiv \tau_{2} \rightarrow \tau_{3} \quad \operatorname{Dom}(\Delta)=\operatorname{Fv}(P) \\
& \frac{\Gamma \vdash_{\mathrm{A}} A: \tau_{1} \quad \Gamma \vdash_{\mathrm{A}} B: \tau_{2}}{\Gamma \vdash_{\mathrm{A}} A B: \tau_{3}} \text { (Term.Appl) } \frac{\Gamma, \Delta \vdash_{\mathrm{P}} P: \tau_{1} \quad \Gamma, \Delta \vdash_{\mathrm{A}} A: \tau_{2}}{\Gamma \vdash_{\mathrm{A}} P \rightarrow \Delta A: \tau_{1} \rightarrow \tau_{2}} \text { (Term·Abs) }
\end{aligned}
$$

Fig. 8. Well-formed pattern and terms.

structures), we need the partial function arr on types, which transforms a product type into a function type:

$$
\begin{aligned}
& \operatorname{arr}\left(\tau_{1} \rightarrow \tau_{2}\right) \triangleq \tau_{1} \rightarrow \tau_{2} \\
& \operatorname{arr}\left(\tau_{1} \wedge \tau_{2}\right) \triangleq \tau_{3} \rightarrow\left(\tau_{4} \wedge \tau_{5}\right) \begin{cases}\text { if } & \operatorname{arr}\left(\tau_{1}\right) \equiv \tau_{3} \rightarrow \tau_{4} \\
\text { and } & \operatorname{arr}\left(\tau_{2}\right) \equiv \tau_{3} \rightarrow \tau_{5} .\end{cases}
\end{aligned}
$$

Hence, the type system of iRho derives judgments with the shape

$$
\begin{array}{llll}
\Gamma \vdash_{\Gamma} o k & \Gamma \vdash_{\tau} \tau: o k & \Gamma \vdash_{\mathrm{V}} A_{\mathrm{V}}: \tau & \\
\Gamma \vdash_{\rho} \rho: \Gamma^{\prime} & \Gamma \vdash_{\sigma} \sigma: \Gamma^{\prime} & \Gamma \vdash_{\mathrm{P}} P: \tau & \Gamma \vdash_{\mathrm{A}} A: \tau,
\end{array}
$$

which denote well-typed contexts, types, values, environments, stores, patterns and terms, respectively. In the following, we let the symbol $\alpha$ range over $\mathscr{X} \cup \mathscr{K}$. The typing rules for patterns and terms are presented in Figure 8. Note that the following rule is admissible. It says that if $A$ is a pointer to an object of type $\tau$, then its access in memory, denoted by ! $A$, has type $\tau$.

$$
\frac{\Gamma \vdash_{\mathrm{A}} A: \tau \text { ref }}{\Gamma \vdash_{\mathrm{A}} ! A: \tau}(\text { Term } \cdot \text { Deref })
$$

The following descriptions cover the most interesting type-checking rules: 
- (Patt-Start) and (Term.Start) fetch from the context the correct type of variables and constants, respectively.

- (Patt-Struct) and (Term:Struct) assign a product type to a structure that records the type of both elements.

- (Patt·Algbr) and (Term. Appl) deal with application. We will just discuss the term-term application, the pattern-pattern application being similar. The application rule is what one expects for an algorithmic version of a type system; note that before applying terms, we need to transform the type $\tau_{1}$ of $A$ into an arrow type since it could happen that $A$ is a structure containing more branches of the same domain type. The subject of the statement is $a \bar{P} P$ since $\bar{P}$ can be empty and, as usual, application associates to the left.

- (Term.Abs) has the context $\Delta$ added to its premises using the decidable function $\operatorname{Fv}(P)$; the context $\Gamma$ gives types only for algebraic constants.

- (Term.Assign) deals with assignment: the only possible choice is to assign to an expression $A$ of type $\tau$ ref, an object $B$ of type $\tau$.

- (Term.Ref) says that if an object $A$ has type $\tau$, then a pointer to this object, denoted by ref $A$, has type $\tau$ ref.

\subsection{Extra typing rules}

The presentation of the type system is completed by five complementary judgments with the shape

$$
\Gamma \vdash_{\Gamma} o k \text {, or } \Gamma \vdash_{\tau} \tau: o k \text {, or } \Gamma \vdash_{\vee} A_{\vee}: \tau \text { and } \Gamma \vdash_{\rho} \rho: \Gamma^{\prime} \text { or } \Gamma \vdash_{\sigma} \sigma: \Gamma^{\prime}
$$

denoting well-formed contexts, types, values, environments and stores, respectively. These judgments are required when we encode iRho in the Logical Framework of Coq. The type rules of these five new judgments are really much more intuitive and do not need any particular comment. It is worth noting that rule (Value-Algbr) also needs a transformation step for product types into arrow types. The (Value.Clos) and (Value-Fail) rules are also interesting since the inferred type for the environment $\rho$ is 'charged' into the derivation for the pattern abstraction. All of the extra rules are listed in Figure 10.

\section{Metatheory}

In this section we present the main properties of iRho, namely:

1 Our natural semantics for $\Downarrow_{\text {val }}$ is deterministic.

2 Type checking with $\vdash_{A}$ is unique.

3 Subject reduction holds (that is, types are preserved under reduction).

4 Type soundness holds (that is, the type system preserves the evaluator from 'stuck' states).

5 Both type checking and type reconstruction are decidable.

The most crucial proofs have been carried out using a mechanical development with the proof assistant Coq (Liquori and Serpette 2005). Some of this development is presented in detail in Section 7. We start with a natural definition of free variables. 


\section{Context type rules}

$$
\begin{aligned}
& \overline{\emptyset \vdash_{\Gamma} o k}(\mathrm{Ctx} \cdot \text { Axiom }) \quad \frac{\Gamma \vdash_{\Gamma} o k \quad \mathrm{~b} \notin \operatorname{Dom}(\Gamma)}{\Gamma, \mathrm{b}: o k \vdash_{\Gamma} o k} \text { (Ctx.Type) } \\
& \frac{\chi \notin \operatorname{Dom}(\Gamma) \quad \Gamma \vdash_{\tau} \tau: o k \quad \Gamma \vdash_{\Gamma} o k}{\Gamma, \chi: \tau \vdash_{\Gamma} o k}(\mathrm{Ctx} \cdot \operatorname{Var} / \text { Const })
\end{aligned}
$$

\section{Term type rules}

$$
\begin{array}{ll}
\frac{\Gamma_{1}, \mathrm{~b}: o k, \Gamma_{2} \vdash_{\Gamma} o k}{\Gamma_{1}, \mathrm{~b}: o k, \Gamma_{2} \vdash_{\tau} \mathrm{b}: o k} \text { (Type-Start) } & \frac{\Gamma \vdash_{\tau} \tau: o k}{\Gamma \vdash_{\tau} \tau \text { ref }: o k} \text { (Type·Ref) } \\
\frac{\Gamma \vdash_{\tau} \tau_{1}: o k \quad \Gamma \vdash_{\tau} \tau_{2}: o k}{\Gamma \vdash_{\tau} \tau_{1} \rightarrow \tau_{2}: o k} \text { (Type·Arrow) } & \frac{\Gamma \vdash_{\tau} \tau_{1}: o k \quad \Gamma \vdash_{\tau} \tau_{2}: o k}{\Gamma \vdash_{\tau} \tau_{1} \wedge \tau_{2}: o k} \text { (Type-Struct) }
\end{array}
$$

\section{Value type rules}

$\frac{\Gamma_{1}, a: \tau, \Gamma_{2} \vdash_{\Gamma} o k}{(\text { Value.Start }}{ }_{1}$ )

$\Gamma_{1}, a: \tau, \Gamma_{2} \vdash_{\mathrm{v}} a: \tau$

$\frac{\Gamma_{1}, \iota: \tau \text { ref }, \Gamma_{2} \vdash_{\Gamma} o k}{\Gamma_{1}, \iota: \tau \text { ref }, \Gamma_{2} \vdash_{\mathrm{v}} \iota: \tau \text { ref }}\left(\right.$ Value.Start $\left.{ }_{2}\right)$

$\frac{\Gamma \vdash_{\mathrm{v}} A_{\mathrm{v}}: \tau_{1} \quad \Gamma \vdash_{\mathrm{v}} B_{\mathrm{v}}: \tau_{2}}{\Gamma \vdash_{\mathrm{v}} A_{\mathrm{v}}, B_{\mathrm{v}}: \tau_{1} \wedge \tau_{2}}$ (Value.Struct)

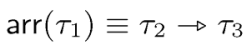

$\Gamma \vdash_{\vee} a \bar{A}_{\vee}: \tau_{1} \quad \Gamma \vdash B_{\mathrm{v}}: \tau_{2}$

$$
\Gamma \vdash_{\mathrm{v}} a \bar{A}_{\mathrm{v}} B_{\mathrm{v}}: \tau_{3}
$$

$\Gamma \vdash \rho \rho: \Gamma^{\prime}$

$\Gamma \vdash_{\mathrm{v}} A_{\mathrm{v}}: \tau_{1} \quad \Gamma \vdash_{\rho} \rho: \Gamma^{\prime}$

$\Gamma^{\prime} \vdash_{\mathrm{p}} P \rightarrow \Delta A: \tau_{1} \rightarrow \tau_{2}$

$\Gamma^{\prime} \vdash_{\mathrm{P}} P \rightarrow \Delta B: \tau_{1} \rightarrow \tau_{2}$

$\Gamma \vdash_{\mathrm{v}}\langle P \rightarrow \Delta A \cdot \rho\rangle: \tau_{1} \rightarrow \tau_{2}$

$\Gamma \vdash_{\mathrm{v}}\left\langle\left[P \ll \Delta A_{\mathrm{v}}\right] . B \cdot \rho\right\rangle: \tau_{2}$

\section{Store type rules}

\section{Environment type rules}

$$
\begin{aligned}
& \Gamma[\iota: \tau] \triangleq\left\{\begin{array}{cc}
\Gamma, \iota: \tau & \text { if } \iota \notin \operatorname{Dom}(\Gamma) \\
\Gamma & \text { if } \iota: \tau \in \Gamma
\end{array}\right. \\
& \frac{\Gamma \vdash_{\Gamma} o k}{\Gamma \vdash_{\sigma} \emptyset: \Gamma} \text { (Store·Axiom) } \\
& \Gamma \vdash_{\sigma} \sigma: \Gamma^{\prime} \quad \Gamma^{\prime}[\iota: \tau] \vdash_{\Gamma} o k \\
& \Gamma \vdash_{\mathrm{v}} \iota: \tau \text { ref } \quad \Gamma \vdash_{\mathrm{v}} A_{\mathrm{v}}: \tau \\
& \Gamma \vdash_{\sigma} \sigma\left[\iota \mapsto A_{\mathrm{v}}\right]: \Gamma^{\prime}[\iota: \tau]
\end{aligned}
$$$$
\Gamma[X: \tau] \triangleq \begin{cases}\Gamma, X: \tau & \text { if } X \notin \operatorname{Dom}(\Gamma) \\ \Gamma & \text { if } X: \tau \in \Gamma\end{cases}
$$$$
\frac{\Gamma \vdash_{\Gamma} o k}{\Gamma \vdash_{\rho} \emptyset: \Gamma}(\text { Env } \cdot \text { Axiom })
$$$$
\Gamma \vdash_{\rho} \rho: \Gamma^{\prime} \quad \Gamma^{\prime}[X: \tau] \vdash_{\Gamma} o k
$$$$
\Gamma \vdash_{\mathrm{A}} X: \tau \quad \Gamma \vdash_{\mathrm{v}} A_{\mathrm{v}}: \tau
$$$$
\Gamma \vdash_{\rho} \rho\left[X \mapsto A_{\mathrm{v}}\right]: \Gamma^{\prime}[X: \tau]
$$

Fig. 9. Extra typing rules. 
Definition 1 (Free variables Fv ).

$$
\begin{array}{llll}
\mathrm{Fv}(a) & \triangleq \varnothing & \mathrm{Fv}(P \rightarrow \Delta A) \triangleq \mathrm{Fv}(A) \backslash \mathrm{Fv}(P) \\
\mathrm{Fv}(X) \triangleq\{X\} & \mathrm{Fv}(A B) & \triangleq \mathrm{Fv}(A) \cup \mathrm{Fv}(B) \\
\mathrm{Fv}(! A) \triangleq \operatorname{Fv}(A) & \mathrm{Fv}(A, B) & \triangleq \mathrm{Fv}(A) \cup \mathrm{Fv}(B) \\
\mathrm{Fv}(\operatorname{ref} A) \triangleq \mathrm{Fv}(A) & \mathrm{Fv}(A:=B) \triangleq \mathrm{Fv}(A) \cup \mathrm{Fv}(B) .
\end{array}
$$

Now we prove that our natural semantics is deterministic.

Theorem 2 (Deterministic semantics). For any term $A$, environment $\rho$ and store $\sigma$ :

1 If $\sigma_{1} \cdot \rho \vdash A \Downarrow_{\mathrm{val}} A_{\mathrm{v}} \cdot \sigma_{2}$ and $\sigma_{1} \cdot \rho \vdash A \Downarrow_{\mathrm{val}} B_{\mathrm{v}} \cdot \sigma_{3}$, then $A_{\mathrm{v}} \equiv B_{\mathrm{v}}$ and $\sigma_{2} \equiv \sigma_{3}$.

2 If $\sigma_{1} \vdash\left\langle A_{\mathrm{v}} \cdot B_{\mathrm{v}}\right\rangle \Downarrow_{\text {call }} C_{\mathrm{v}} \cdot \sigma_{2}$ and $\sigma_{1} \vdash\left\langle A_{\mathrm{v}} \cdot B_{\mathrm{v}}\right\rangle \Downarrow_{\text {call }} D_{\mathrm{v}} \cdot \sigma_{3}$, then $C_{\mathrm{v}} \equiv D_{\mathrm{v}}$ and $\sigma_{2} \equiv \sigma_{3}$.

3 If $\sigma \cdot \rho_{1} \vdash\left\langle P . A_{\mathrm{v}}\right\rangle \Downarrow_{\text {match }} \rho_{2}$ and $\sigma \cdot \rho_{1} \vdash\left\langle P . A_{\mathrm{v}}\right\rangle \Downarrow_{\text {match }} \rho_{3}$, then $\rho_{2} \equiv \rho_{3}$.

Proof. The theorem was proved using Coq.

The type system presentation is syntax directed, so it suggests directly how we can build an algorithm, and thus that it enjoys the nice property of uniqueness of typing; a software prototype of a simple type checker can be found in the web appendix (Liquori and Serpette 2005)).

Theorem 3 (Uniqueness of typing). If $\Gamma \vdash_{\mathrm{A}} A: \tau_{1}$, and $\Gamma \vdash_{\mathrm{A}} A: \tau_{2}$, then $\tau_{1} \equiv \tau_{2}$.

Proof. The theorem was proved using Coq.

The following definition splits the typed context into two sub-contexts: the former recording types assigned to locations, and the latter recording types assigned to variables. This will be useful in the Subject-reduction Theorem.

Definition 4 (Coherence). The context $\Gamma$ is coherent with a store $\sigma$, and an environment $\rho$, denoted by

$$
\Gamma \vdash \operatorname{coh} \sigma \cdot \rho
$$

if there exist two sub-contexts $\Gamma_{1}$ and $\Gamma_{2}$ such that $\Gamma_{1}, \Gamma_{2} \equiv \Gamma$ and $\Gamma_{1} \vdash_{\sigma} \sigma: \Gamma_{1}$ and $\Gamma_{2} \vdash_{\rho} \rho: \Gamma_{2}$.

Proving subject reduction for open terms is preliminary to proving it for closed terms.

Theorem 5 (Subject reduction for open terms). If $\sigma_{1} \cdot \rho_{1} \vdash A \Downarrow_{\text {val }} A_{\mathrm{v}} \cdot \sigma_{2}$ and $\Gamma_{1} \vdash_{\text {coh }} \sigma_{1} \cdot \rho_{1}$ and $\Gamma_{1} \vdash_{\mathrm{A}} A: \tau$, then there exists $\Gamma_{2}$, which extends $\Gamma_{1}$, such that $\Gamma_{2} \vdash_{\text {coh }} \sigma_{2} \cdot \rho_{1}$ and $\Gamma_{2} \vdash_{\mathrm{v}} A_{\mathrm{v}}: \tau$.

Proof. The theorem was proved using Coq.

The following result is crucial for type soundness.

Theorem 6 (Subject reduction for closed terms). If $\varnothing . \varnothing \vdash A \Downarrow_{\mathrm{val}} A_{\mathrm{v}} \cdot \sigma$ and $\varnothing \vdash_{\mathrm{A}} A: \tau$, then there exists $\Gamma$ such that $\Gamma \vdash_{\text {coh }} \sigma . \varnothing$ and $\Gamma \vdash_{\mathrm{v}} A_{\mathrm{v}}: \tau$.

Proof. The theorem was proved using Coq. 
The reduction rules for the operational semantics given in Figure 6 readily suggest how an interpreter for iRho can be defined. Run-time errors for this interpreter would correspond to stuck states when using the rules to evaluate a closed expression. An inspection of the three judgments of the operational semantics shows that there are only two ways in which an evaluation may get 'stuck':

- $\left(\Downarrow_{\text {val }}\right)$ gets stuck when we access a variable not defined in the environment, when the evaluation of $A$ in $A:=B$ gives a fresh, dangling location (that is, one not in the current used store), or if a premise in some judgment gets stuck.

- $\left(\Downarrow_{\text {call }}\right)$ gets stuck when we try to apply a location value to a value (for example, $\left\langle\imath \cdot A_{\mathrm{v}}\right\rangle$ ), or if a premise in some judgment gets stuck.

- $\left(\Downarrow_{\text {match }}\right)$ gets stuck when we try to match a pattern against a value with a different (unmatchable) shape, for example, $\langle(P Q)$. $(A, B)\rangle$.

The following soundness theorem proves the absence of such errors in the evaluation of a well-typed closed expression.

Theorem 7 (Progress and type soundness). Let $A$ be a closed term such that $\varnothing \vdash_{\mathrm{A}} A: \tau$ is derivable, and let $\mathrm{C}[\cdot]$ be any iRho-context.

\section{Progress}

$\left(\Downarrow_{\text {val }}\right)$

1 If $A \equiv \mathrm{C}[X]$, there exist $\sigma_{1}$ and $\rho$ such that $\sigma_{1} \cdot \rho \vdash X \Downarrow_{\text {val }} \rho(X) \cdot \sigma_{2}$ and $\rho(X) \neq \perp$.

2 If $A \equiv \mathrm{C}[B:=C]$, there exist $\sigma_{1}$ and $\rho$ such that $\sigma_{1} . \rho \vdash B \Downarrow_{\text {val }} l \cdot \sigma_{2}$ and $\imath \in \operatorname{Dom}\left(\sigma_{2}\right)$.

( $\left.\Downarrow_{\text {call }}\right)$

If $A \equiv \mathrm{C}[B C]$, there exist $\sigma_{1}$ and $\rho$ such that $\sigma_{1} \cdot \rho \vdash B \Downarrow_{\mathrm{val}} B_{\mathrm{v}} \cdot \sigma_{2}$, and $B_{\mathrm{v}} \not \equiv \imath$;

$\left(\Downarrow_{\text {match }}\right)$

If $A \equiv \mathrm{C}[B C]$, there exist $\sigma_{1}$ and $\rho$ such that $\sigma_{1} \cdot \rho \vdash B \Downarrow_{\text {val }}\left\langle P \rightarrow_{\Delta} D \cdot \rho_{1}\right\rangle \cdot \sigma_{2}$ and $\sigma_{2} \cdot \rho \vdash C \Downarrow_{\text {val }} C_{\mathrm{v}} \cdot \sigma_{3}$, and $P$ will successfully match with $C_{\mathrm{v}}$.

\section{Type-soundness}

If $\varnothing \vdash_{\mathrm{A}} A: \tau$, then $\varnothing . \varnothing \vdash A \Downarrow_{\mathrm{val}} A_{\mathrm{v}}$ for some $A_{\mathrm{v}}$.

Proof.

\section{Progress}

( $\left.\Downarrow_{\text {val }}\right)$ If $A$ has a variable $X$ as a sub-expression, then, by the well-typedness of the sub-expressions, environment and store, $\rho(X) \neq \perp$. If $A$ has an assignment $B:=C$ as a sub-expression, then, by the well-typedness of the sub-expressions, environment and store, $B$ evaluates to $l \cdot \sigma_{2}$ and $l \in \operatorname{Dom}\left(\sigma_{2}\right)$;

( $\left.\Downarrow_{\text {call }}\right)$ Similarly, if $A$ has an application $B C$ as a sub-expression, then, by the welltypedness of the sub-expressions, environment and store, the evaluation of $B$ is not a location.

( $\left.\Downarrow_{\text {match }}\right)$ Again, if $A$ has an application $B C$ as a sub-expression, then, by the welltypedness of the sub-expressions, environment and store, the evaluation of $B$ leads to a closure value $\left\langle P \rightarrow_{\Delta} D . \rho_{1}\right\rangle$, and the pattern $P$ has a shape that can overlap with $C_{\mathrm{v}}$ (the latter being obtained by the evaluation of $C$ ). 


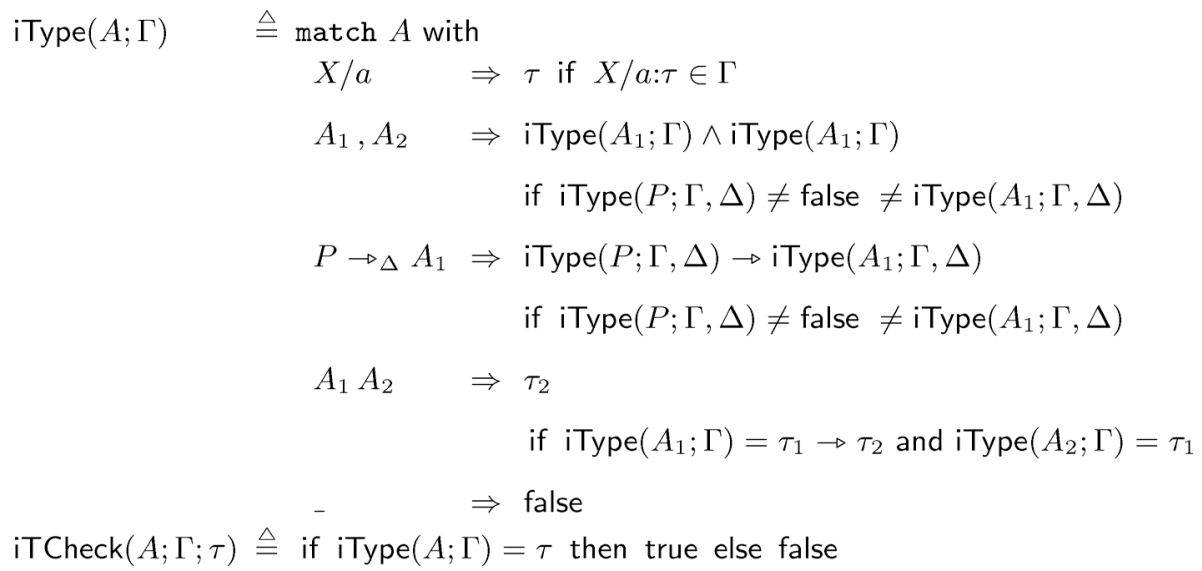

Fig. 10. The algorithms iType and iTCheck.

\section{Type soundness}

Type soundness follows immediately from Progress and the Subject-reduction Theorem.

We conclude this section with some decidability results.

Theorem 8. Given a closed expression $A$, the following propositions are decidable:

1 (Type checking) The existence of a type $\tau$ such that $\varnothing \vdash_{\mathrm{A}} A: \tau$ is decidable.

2 (Type reconstruction) The truth of $\varnothing \vdash_{\mathrm{A}} A: \tau$ for a given $\tau$ is decidable.

Proof.

1 Figure 10 gives the sketch of a recursive algorithm for building $\tau$, or returning false if it does not exist.

2 We use the previous algorithm for type reconstruction (Figure 10). By the uniqueness of typing, $\Gamma \vdash_{\mathrm{A}} A: \tau$ if and only if $\tau$ is equivalent to the type found for $A$.

Theorem 9 (Soundness and completeness of iType). For a closed $A$ and a given $\Delta$, we have iType $(\varepsilon ; A)=\tau$ if and only if $\varepsilon \vdash_{\mathrm{A}} A: \tau$ is derivable.

Proof. It is easy to prove both parts using induction on the structure of $A$.

\section{Examples}

To simplify the derivations, some types are omitted. The first example type checks the imperative term of Example 2. The second example deals with structures and normalised types. The third example evaluates a more complicated imperative term, and the final example provides static and dynamic descriptions of a simple functional fixed point. When no ambiguity arises, we use the following syntactic sugar for multiple assignments:

$$
\left(X_{1} ; \ldots ; X_{n}\right):=\left(A_{1} ; \ldots ; A_{n}\right) \triangleq X_{1}:=A_{1} ; \ldots ; X_{n}:=A_{n} .
$$




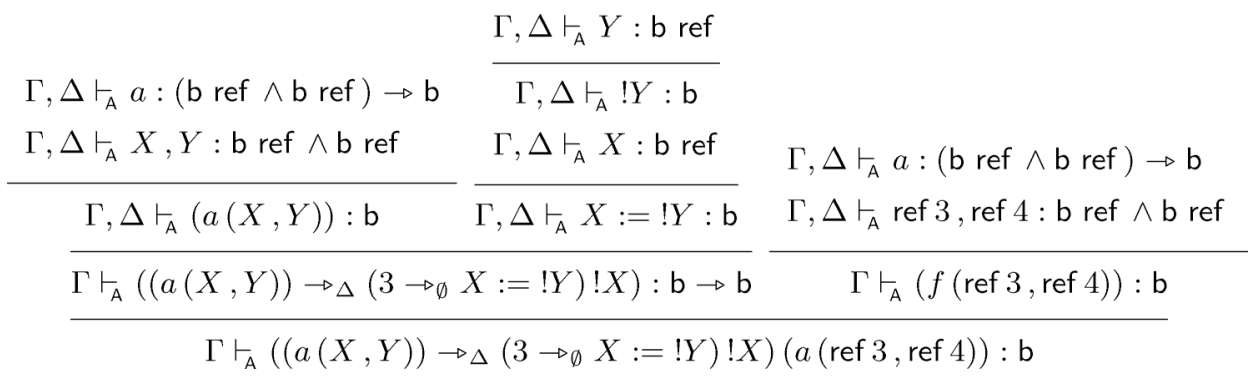

Fig. 11. Type checking of Example 2.

$\Gamma, \Delta \vdash_{\mathrm{A}} X: \mathrm{b}$ ref
$\frac{\Gamma, \Delta \vdash_{\mathrm{A}} Y: \mathrm{b} \text { ref }}{\Gamma, \Delta \vdash_{\mathrm{A}} X, ! Y: \mathrm{b} \text { ref } \wedge \mathrm{b} \text { ref }} \frac{\Gamma, \Delta \vdash_{\mathrm{A}} ! Y: \mathrm{b}}{\Gamma, \Delta \vdash_{\mathrm{A}} X:=! Y: \mathrm{b}} \frac{\begin{array}{c}\Gamma, \Delta \vdash_{\mathrm{A}} X: \mathrm{b} \text { ref } \\ \Gamma, \Delta \vdash_{\mathrm{A}} Y: \mathrm{b} \text { ref }\end{array}}{\frac{\Gamma, \Delta \vdash_{\mathrm{A}} X, Y: \mathrm{b} \text { ref } \wedge \mathrm{b} \text { ref }}{\Gamma \vdash_{\mathrm{A}}(X, Y) \rightarrow \Delta X: \Delta \vdash_{\mathrm{A}} Y: \mathrm{b} \text { ref }}}$
$\frac{\Gamma \vdash_{\mathrm{A}}((X, Y) \rightarrow \Delta X:=! Y,(\mathrm{~b} \text { ref } \wedge \mathrm{b} \text { ref }) \rightarrow \mathrm{b}}{\Gamma \vdash_{\mathrm{A}}(X, Y) \rightarrow \Delta Y:(\mathrm{b} \text { ref } \wedge \mathrm{b} \text { ref }) \rightarrow \mathrm{b}}$
$\operatorname{arr}((\mathrm{b}$ ref $\wedge \mathrm{b}$ ref $) \rightarrow \mathrm{b} \wedge(\mathrm{b}$ ref $\wedge \mathrm{b}$ ref $) \rightarrow \mathrm{b}) \equiv(\mathrm{b}$ ref $\wedge \mathrm{b}$ ref $) \rightarrow(\mathrm{b} \wedge \mathrm{b})$
$\Gamma \vdash_{\mathrm{A}}(\operatorname{ref} a, \operatorname{ref} b): \mathrm{b}$ ref $\wedge \mathrm{b}$ ref

$$
\Gamma \vdash_{\mathrm{A}}((X, Y) \rightarrow \Delta X:=! Y,(X, Y) \rightarrow \Delta ! Y)(\operatorname{ref} a, \operatorname{ref} b): \mathrm{b} \wedge \mathrm{b}
$$

Fig. 12. Type checking of $\left((X, Y) \rightarrow_{\Delta} X:=! Y,(X, Y) \rightarrow_{\Delta} Y\right)(a, b)$.

\subsection{Basic examples}

Example 3 (Type checking). The type checking of Example 2 is shown in Figure 11, where $\Gamma \equiv \mathrm{b}: o k, 3: \mathrm{b}, 4: \mathrm{b}, a:(\mathrm{b}$ ref $\wedge \mathrm{b}$ ref $) \rightarrow \mathrm{b}$ and $\Delta \equiv X: \mathrm{b}$ ref, $Y: \mathrm{b}$ ref .

Example 4 (Structures and normalised types). Let $\Gamma \equiv \mathrm{b}: o k, a: \mathrm{b}, b: \mathrm{b}$ and $\Delta \equiv X: \mathrm{b}$ ref, $Y$ : b ref. A derivation for

$$
\left((X, Y) \rightarrow_{\Delta} X:=! Y \quad,(X, Y) \rightarrow_{\Delta} Y\right)(a, b)
$$

is shown in Figure 12.

\subsection{Some trickier examples}

Example 5 (Negation normal form). This function (computing a negation normal form) is used in implementing decision procedures, which are present in almost all model checkers. The processed input is an implication-free language of formulas with generating grammar

$$
\phi::=\mathrm{p} \mid(\text { and }(\phi, \phi))|(\operatorname{or}(\phi, \phi))|(\operatorname{not} \phi) .
$$

We present two imperative encodings: in the first, the function is shared using a pointer, and recursion is achieved through dereferencing. In the second, formulas are again shared, 


$$
\begin{aligned}
& n n f 1 \triangleq\left(\begin{array}{lll}
\mathrm{p} & \rightarrow \mathrm{p}, \\
(\operatorname{not}(\operatorname{not} X)) & \rightarrow !(\operatorname{SELFX}), \\
(\operatorname{not}(\operatorname{or}(\mathrm{X}, \mathrm{Y}))) & \rightarrow & (\text { and }(!(\operatorname{SELF}(\operatorname{not} X)))), !(\operatorname{SELF}(\operatorname{not} Y)), \\
(\operatorname{not}(\operatorname{and}(X, Y))) & \rightarrow & (\operatorname{or}(!(\operatorname{SELF}(\operatorname{not} X)))), !(\operatorname{SELF}(\operatorname{not} Y)), \\
(\operatorname{and}(X, Y)) & \rightarrow & (\operatorname{and} !(\operatorname{SELF} X), !(\operatorname{SELFY})), \\
(\operatorname{or}(X, Y)) & \rightarrow & (\operatorname{or}(!(\operatorname{SELF} X), !(\operatorname{SELF} Y)))
\end{array}\right)
\end{aligned}
$$

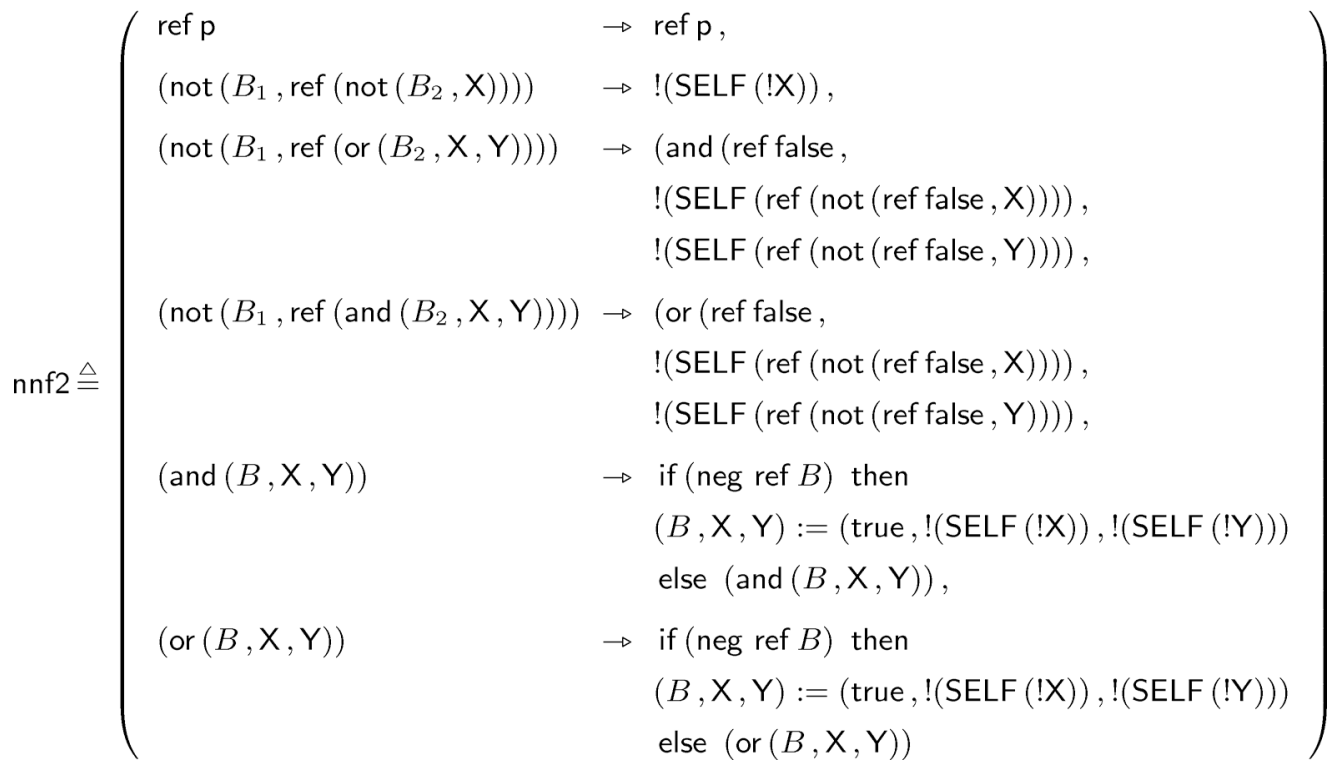

Fig. 13. Imperative encoding with(out) sharing.

through back-pointers to shared sub-trees. The variable 'SELF' plays the role of the metavariable 'self' (or 'this') commonly found in object-oriented languages. Then we type check the encodings. For the sake of readability, all type decorations within terms are omitted.

\section{Imperative (I)}

This encoding uses a variable SELF, which contains a pointer to the recursive code: here the recursion is achieved directly through pointer dereferencing, assignment and the classical imperative fixed point in order to implement recursion. Given the constant dummy, the function nnf1 is defined as in Figure 13, and the imperative encoding is

$$
\text { let } \mathrm{SELF} \ll \text { ref dummy in let NNF } \ll \text { nnf1 in SELF :=NNF; (NNF } \phi) \text {. }
$$

\section{Imperative with sharing (IS)}

This encoding uses a variable SELF, which contains a pointer to the recursive code and a flag pointer to a boolean value associated with each node: all flag pointers are initially set to false; each time we scan a (possibly) shared formulas we set the 


$$
\begin{aligned}
& \Gamma, \Delta \vdash_{\mathrm{A}} \text { fix }:(\mathrm{b} \rightarrow \mathrm{b}) \rightarrow \mathrm{b} \quad \Gamma, \Delta \vdash_{\mathrm{A}} X: \mathrm{b} \rightarrow \mathrm{b} \\
& \Gamma, \Delta \vdash_{\mathrm{A}} X: \mathrm{b} \rightarrow \mathrm{b} \quad \Gamma, \Delta \vdash_{\mathrm{A}}(\text { fix } X): \mathrm{b} \quad \Gamma, \Delta \vdash_{\mathrm{A}} \text { fix }:(\mathrm{b} \rightarrow \mathrm{b}) \rightarrow \mathrm{b} \\
& \frac{\Gamma, \Delta \vdash_{\mathrm{A}}(\mathrm{fix} X): \mathrm{b}}{\Gamma, \Delta \vdash_{\mathrm{A}} X(\mathrm{fix} X): \mathrm{b}} \frac{\Gamma, \Delta \vdash_{\mathrm{A}} \Omega: \mathrm{b} \rightarrow \mathrm{b}}{\Gamma, \Delta \vdash_{\mathrm{A}}(\mathrm{fix} \Omega): \mathrm{b}} \\
& \Gamma \vdash_{\mathrm{A}} \Omega(\mathrm{fix} \Omega): \mathbf{b} \\
& \frac{\infty}{\emptyset \vdash \Omega(\text { fix } \Omega) \Downarrow_{\text {val }} \text { segmentation fault }}
\end{aligned}
$$

Fig. 14. One fixed point.

corresponding flag pointer to true. The grammar of shared formulas is

$$
\begin{array}{ll}
\text { bool } & :=\text { true } \mid \text { false } \\
\text { flag } & ::=\text { bool ref } \\
\psi & ::=\operatorname{ref} \phi \\
\phi & ::=\mathrm{p} \mid(\text { and }(\text { flag }, \psi, \psi)) \mid(\operatorname{or}(\text { flag }, \psi, \psi)) \mid(\operatorname{not}(\text { flag }, \psi)) .
\end{array}
$$

Given the constant dummy, the function nnf2 is defined as in Figure 13, and the imperative encoding is

let SELF $\ll$ ref dummy in let $\mathrm{NNF} \ll \mathrm{nnf} 2$ in SELF $:=\mathrm{NNF} ;(\mathrm{NNF} \psi)$.

\section{Typing the imperative encodings}

If $\mathbf{b}$ is the type of formulas $\phi$ and $\mathbf{b}$ ref is the type of the shared formulas $\psi$, and $\wedge \tau \triangleq \underbrace{\tau \wedge \ldots \wedge \tau}_{n}$ and $\tau_{1} \triangleq \mathrm{b} \rightarrow \mathrm{b}$ and $\tau_{2} \triangleq \mathrm{b}$ ref $\rightarrow \mathrm{b}$ ref, then it is easy to verify that the following judgments are derivable (we let $\Gamma_{1} \triangleq$ dummy: $\wedge^{6} \tau_{1}, \operatorname{SELF}: \wedge^{\varsigma} \tau_{1}$ ref and $\Gamma_{2} \triangleq$ dummy: $\wedge^{6} \tau_{2}$, SELF $: \wedge^{6} \tau_{2}$ ref $):$

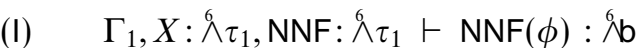

$$
\begin{aligned}
& \text { (IS) } \quad \Gamma_{2}, X: \AA^{6} \tau_{2}, \mathrm{NNF}: \wedge^{6} \tau_{2} \vdash \operatorname{NNF}(\psi): \AA^{6} \mathrm{~b} \text { ref. }
\end{aligned}
$$

Example 6 (Simple first-order fixed point (Cirstea et al.) 2004). The type systems of iRho do not obey the classical property that 'well-typed programs normalise'. More precisely, non-termination can be encoded in our calculus thanks to ad hoc patterns. We present here a term inspired by the classical $\Omega$ term of the untyped lambda calculus. Let $\Gamma \equiv \mathrm{fix}:(\mathrm{b} \rightarrow \mathrm{b}) \rightarrow \mathrm{b}$ and $\Delta \equiv X: \mathrm{b} \rightarrow \mathrm{b}$. A derivation for $\Omega \triangleq($ fix $X) \rightarrow \Delta(X($ fix $X))$ is shown in Figure 14. It is easy to verify that our interpreter diverges on this term.

Remark 3 (On let rec and fixed points). Fixed points and let rec definitions are introduced using the well-known result of Nax Paul Mendler (Mendler et al. 1986; Mendler 1987). 
When introducing recursive definitions in the typed lambda calculus, the strong normalisation is no longer enforced by typing if the type constructors do not satisfy a positiveness condition.

This condition forces an algebraic constructor to be typed without negative occurrences of recursive (potentially infinite) entities; in our case, the algebraic constructor fix (see Example 6) involved does not satisfy the above condition since it is applied to a recursive object represented by the SELF variable. This condition is also enforced in the Calculus of Inductive Constructions (see Gimenez (1998)), which is the basis of the Coq proof assistant. The condition avoids inconsistencies in the system itself, such as proving the Russell Paradox; termination issues are essential in Curry-Howard based proof assistants. The same problem also appears in programming languages: for instance, one can define a recursive function in Caml without using the keyword let rec.

There are many techniques for implementing recursive definitions efficiently and effectively in call-by-value functional languages: among them, it is worth noting the 'in-place update tricks' outlined by Guy Cousineau et al. (Cousineau et al. 1987), and the more recent techniques due to Gérard Boudol and Pascal Zimmer (Boudol and Zimmer 2002), and Tom Hirschowitz et al. (Hirschowitz et al. 2003), or Peter Landin's classical trick (Landin 1964).

\section{Formalisation in Coq}

In the previous sections we have given a mathematical presentation of iRho suited to an encoding in Coq. The formalisation of iRho in the specification language of the proof assistant is, however, a complex task as we have to face many subtle details that are left implicit on paper. Here we will just briefly discuss the most interesting aspects of this development.

The encoding of iRho in Coq rephrases the previous sections in a natural way. The adequacy of the Coq encoding with respect to the mathematical presentation is proved using pen and paper.

A well-known problem we have to deal with is the encoding of the $\rightarrow$-binder. Binders are known to be difficult to encode in proof assistants; our encoding was essentially based on closures, that is, <pattern abstraction - environment $>$ pairs. Environments are partial functions from variables to values. Substitution is replaced by a simple look up in the environment; variable scoping, and all name-related matters are simply ignored. This technique is widely used in efficient implementations of functional languages, and greatly simplifies mechanical metatheory.

\subsection{Syntactic and semantic structures}

The signature of the encoding of iRho is presented in Figure 15 . We will only comment on the most interesting choices:

- An ad hoc type var is introduced for variables; the only terms that can inhabit var are the variables in the logical framework. Thus, $\alpha$-equivalence on terms is immediately inherited from the metalanguage, together with induction and recursion principles. 


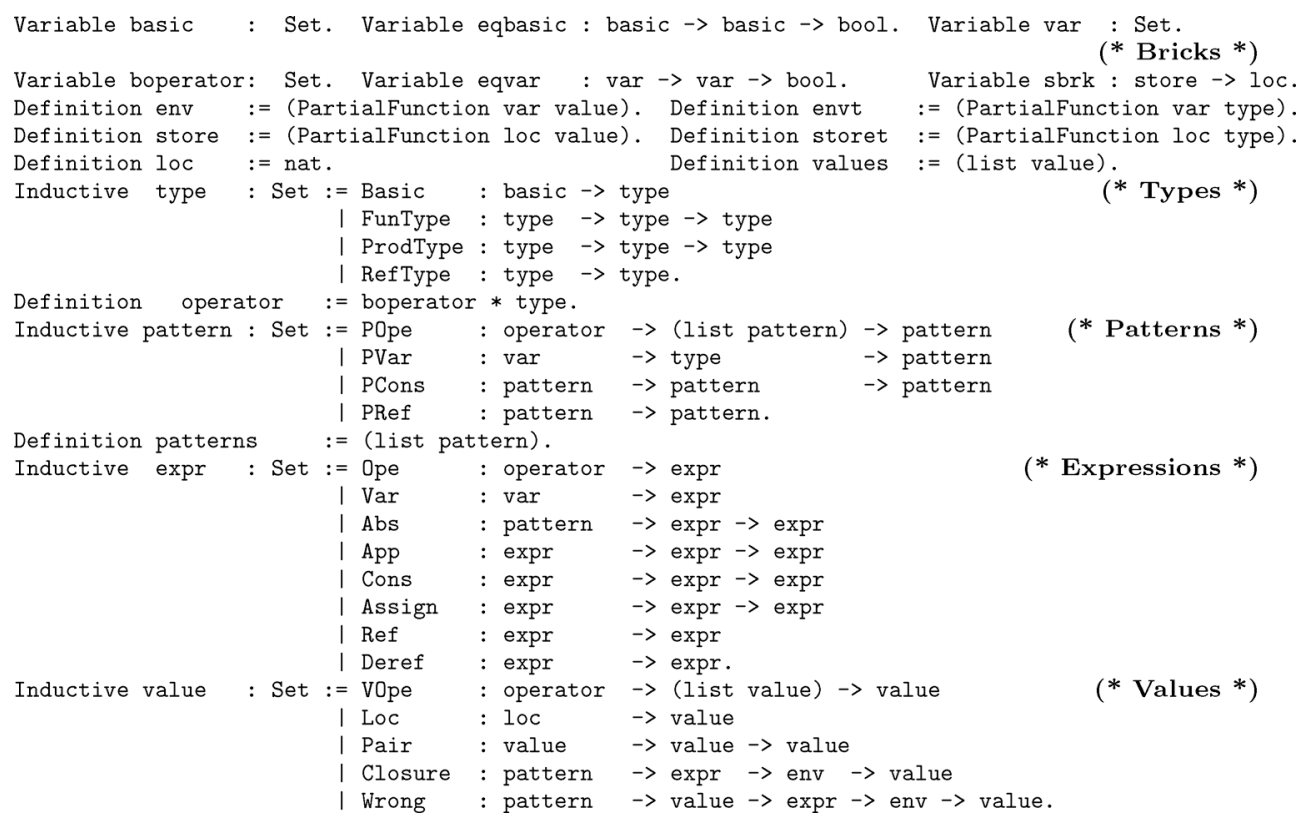

Fig. 15. Semantics domains in Coq.

- Another ad hoc type boperator is introduced for algebraic constants; algebraic constants come together with their types, thereby giving the category operator as a boperator*type pair.

- Locations loc are faithfully represented by natural numbers.

- (Un)typed environments (env and envt) and (un)typed stores (store and storet) are partial functions from var/loc to the sets value/type.

- A special variable sbrk denotes a function that, for any store, gives the topmost unused location: the sbrk variable is essential when we are looking to extend the store with fresh locations during new allocations (using the operator ref).

- Types type needs no special comments: they are implemented with an inductive datatype; patterns pattern, expressions expr, and values value are also implemented by an inductive datatype.

\subsection{Natural semantics}

As we mentioned in the previous section, the natural semantics is given by means of two mutually recursive functions, namely, eval and call, and a third function match devoted to calculate matching; they are sketched in Figure 16. The web appendix (Liquori and Serpette 2005) contains the complete encoding of the natural semantics. None of the rules present any surprises when compared with the rules in the natural semantics (that is, we get adequacy almost directly). We will only comment on the most interesting choices:

- (evalApp) is an 'ASCII-clone' of the (Red·Appl $\left.l_{\sqrt{ }}\right)$ natural semantic rule. 


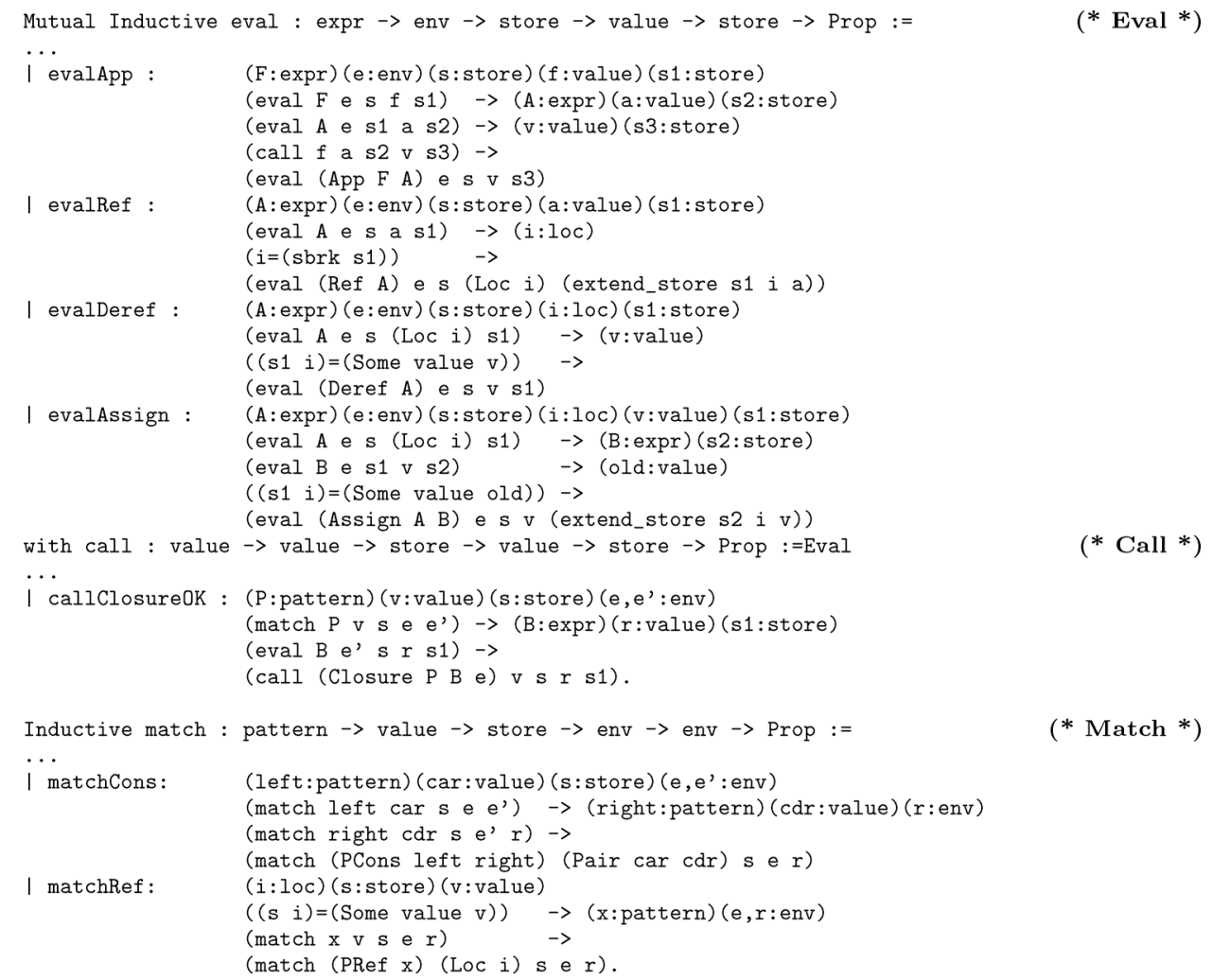

Fig. 16. Sketch of natural imperative semantics in Coq.

- (evalRef) encodes the semantic rule (Red-Ref). Observe the use of the sbrk function, which extends a given store (partial function) through the (here omitted) auxiliary function extend_store.

- (evalDeref) first verifies that the required location belongs to the store domain (recall that stores are partial functions), and then directly accesses the store leaving the store itself unmodified.

- (evalAssign) first evaluates the Ivalue and the rvalue, then verifies that the location corresponds to the Ivalue defined in the store, and finally modifies the store in situ.

- (callclosureOK) and (matchCons) are also just clones of the natural semantic rules (Call-FunOK) and (Match·Pair), respectively.

- (matchRef) first verifies that the given location $i$ has some meaning in the store $s$, and then matches the $x$ pattern in (PRef $x$ ) against (Loc $i$ ).

\subsection{Type system}

The encoding of the type system is rather straightforward. The encoding consists of three inductive functions, namely TypeCheckPattern, TypeCheckExpr and Type0f, to type check patterns, terms and values, respectively. The latter function needs two important auxiliary 


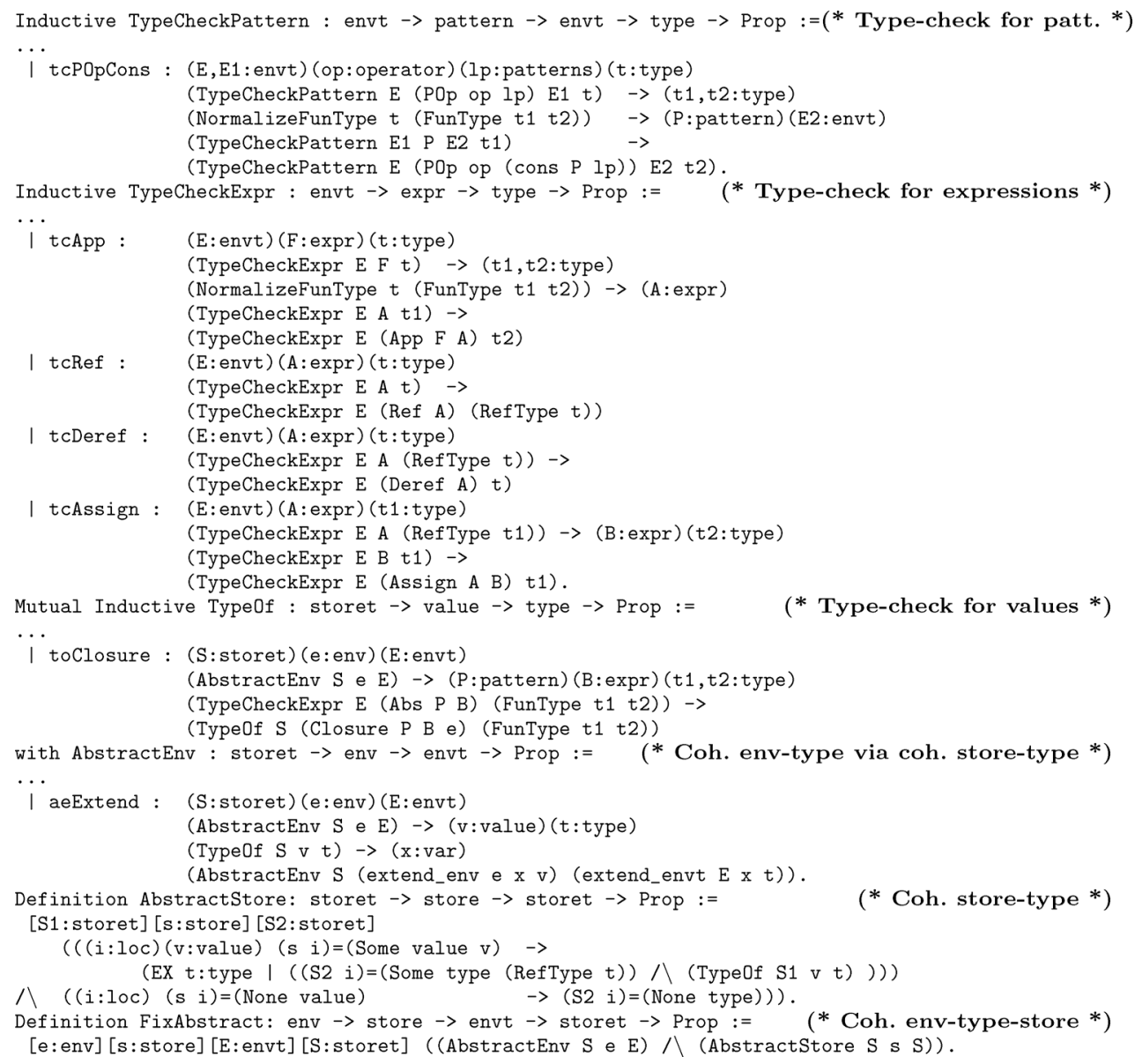

Fig. 17. Sketch of type-checking rules in Coq.

functions, namely AbstractEnv, and AbstractStore, to maintain consistency between types environments $(\Gamma)$ and typed stores $(\sigma)$. We will only discuss the most interesting rules presented in Figure 17:

- (tcPOpCons) and (tcApp) encode the type checking rule for patterns (Patt·Algbr), and terms (Term·Appl), respectively - they make use of the function NormalizeFunType which behaves as a coercion to a functional type.

- (tcRef), (tcDeref) and (tcAssign) encode the type rules (Type-Ref), (Type-Deref) and (Type-Assign), respectively - they just mimic the corresponding rules in the natural semantics.

- (aeExtend) is the counterpart of the judgment $\vdash_{\rho}$ (see Subsection 4.1), which assigns a type (that is, a context) to an untyped environment - to ease the proof development, the encoding makes use of two different partial functions, namely envt and storet, to give a type to untyped environments and store, but this 'nuance' disappears in the typing rule, where a context $\Gamma$ binds variables and/or locations to types; a coherence 
theorem (see Section 5) bridges the gap between the mathematical presentation and the encoding.

- (toClosure) faithfully encodes rule (Value.Clos) - see Subsection 4.1.

- (FixAbstract) is crucial for establishing a coherence relation between (un)typed environments and (un)typed stores.

\subsection{Some metatheory in Coq}

The following theorems collect some results we have proved in Coq on both the dynamic and static semantics: see Section 5 for the the full metatheory, and Liquori and Serpette (2005) for its complete mechanical counterparts.

\section{Theorem 10 (Coq's run-time galleria).}

1 Lemma LowerWhenSbrk : (s:store) (i:loc) $i=($ sbrk $s) \rightarrow$ (a:value) (Lower s (extend_store s i a)).

2 Lemma NoGarbageCollection : (E: expr) (e:env)(s:store)(v:value)(s1:store) (eval E e s v s1) $\rightarrow$ (LowerDomain s s1).

3 Lemma match_deterministic : (P:pattern) (v:value) (s:store) (e:env) (e1:env) (match $\mathrm{P} v \mathrm{~s}$ e e1) $\rightarrow$ (e2:env) (match $P$ V s e e2) $\rightarrow(e 1=e 2)$

4 Theorem eval_deterministic : (A:expr) (e:env) (s:store) (v1:value) (s1:store) (eval A e s v1 s1) $\rightarrow$ (v2:value) (s2:store) (eval A e s v2 s2) $\rightarrow((\mathrm{v} 1=\mathrm{v} 2) /(\mathrm{s} 1=\mathrm{s} 2))$.

5 Theorem call_deterministic: (v1,v2:value)(s:store)( 1 : value)(s1:store) (call v1 v2 s r1 s1) $\rightarrow$ (r2:value) (s2:store) (call v1 v2 $\mathrm{s}$ r2 $\mathrm{s} 2) \rightarrow((\mathrm{r} 1=\mathrm{r} 2) \wedge(\mathrm{s} 1=\mathrm{s} 2))$

\section{Theorem 11 (Coq's compile-time galleria).}

1 Lemma NormalizeFunType deterministic: $(t, t 1: t y p e)$ (NormalizeFunType $t$ t 1$) \rightarrow$ (t2:type) (NormalizeFunType $t$ t2) $\rightarrow(t 1=t 2)$.

2 Lemma TypeCheckPattern_deterministic: (E:envt) (P:pattern) (E1:envt) (t1:type) (TypeCheckPattern E P E1 t1) $\rightarrow$ (E2: envt) (t2:type) (TypeCheckPattern E P E2 t2) $\rightarrow((\mathrm{E} 1=\mathrm{E} 2) \wedge(\mathrm{t} 1=\mathrm{t} 2))$.

3 Lemma TypeCheckExpr_deterministic: (E:envt) (A:expr) (t1:type) (* algo type check expr *) (TypeCheckExpr E A t1) $\rightarrow$ (t2:type) (TypeCheckExpr E A t2) $\rightarrow$ (t1=t2).

4 Lemma open_subject_reduction_match: (P:pattern) (v:value) (s:store) (e,e':env) (match $P$ v s e e') $\rightarrow$ (E:envt) (S:storet) (FixAbstract e $S$ E S) $\quad \rightarrow$ (E1:envt) (t1:type) (TypeCheckPattern E P E1 t1) $\rightarrow$ (TypeOf S v t1) $\rightarrow$ (AbstractEnv S e' E1).

5 Lemma open_subject_reduction: (A: expr) (e:env) (s:store) (v:value) (s2:store) (eval A e s v s2) $\rightarrow$ (E:envt) (S: storet) (FixAbstract e $S$ E $S$ ) $\rightarrow$ (t:type)

(TypeCheckExpr E A t) $\rightarrow$ (EX S2:storet | ((Coherent s S s2 S2) 八 (TypeOf S2 v t))).

6 Theorem subject_reduction: (A:expr) (v:value) (s2:store) (eval A env init store init $v$ s2) $\rightarrow$ (t:type)

(TypeCheckExpr envt_init A t) $\rightarrow$

(EX S2:storet | ((Coherent store_init storet_init s2 S2) $\backslash($ Type0f S2 v t $))$ )).

* SR for open expr match*)

Since the data structures for stores, environments, terms, values and types are isomorphic in 'mathematics' and in Coq, the adequacy result comes directly as a matter of fact. To resume, all theorems in the following list that have been proved by the proof assistant Coqare labelled with a ' $\sqrt{ }$ '.

\section{(Determinism) $\sqrt{ }$}

$$
\text { If } \sigma \cdot \rho \vdash A \Downarrow_{\mathrm{val}} A_{\vee}^{\prime} \cdot \sigma^{\prime} \text { and } \sigma \cdot \rho \vdash A \Downarrow_{\mathrm{val}} A_{\vee}^{\prime \prime} \cdot \sigma^{\prime \prime} \text {, then } A_{\vee}^{\prime} \equiv A_{\vee}^{\prime \prime} \text { and } \sigma^{\prime} \equiv \sigma^{\prime \prime} \text {. }
$$


(Unique Type) $\sqrt{ }$

If $\Gamma \vdash_{\mathrm{A}} A: \tau$, then $\tau$ is unique.

(Coherence) $\sqrt{ }$

$\sigma \cdot \rho \vdash_{\text {coh }} \Gamma$ if there exist two sub-contexts $\Gamma_{1}$ and $\Gamma_{2}$ such that $\Gamma_{1}, \Gamma_{2} \equiv \Gamma$ and $\Gamma \vdash_{\sigma} \sigma: \Gamma_{1}$ and $\Gamma \vdash_{\rho} \rho: \Gamma_{2}$.

\section{(Subject reduction) $\checkmark$}

If $\varnothing \vdash_{\mathrm{A}} A: \tau$ and $\varnothing . \varnothing \vdash A \Downarrow_{\mathrm{val}} A_{\mathrm{v}}, \sigma$, then there exists $\Gamma^{\prime}$ that extends $\Gamma$ such that $\Gamma^{\prime} \vdash_{\sigma} \sigma: o k$ and $\Gamma^{\prime} \vdash_{\mathrm{v}} A_{\mathrm{v}}: \tau$.

\section{(Type soundness)}

If $\varnothing \vdash_{\mathrm{A}} A: \tau$, then $\varnothing . \varnothing \vdash A \Downarrow_{\text {call }} A_{\mathrm{v}}$ for some $A_{\mathrm{v}}$.

\section{(Type checking)}

The existence of a type $\tau$ such that $\varnothing \vdash_{\mathrm{A}} A: \tau$ is decidable.

\section{(Type reconstruction)}

The truth of $\varnothing \vdash_{\mathrm{A}} A: \tau$ for a given $\tau$ is decidable.

\section{Conclusions, related work and future directions}

In this paper we have presented a formal development of the theory of iRho, a typed rewriting-based calculus featuring term rewriting, pattern matching on imperative terms, structures, functions and side effects. We have mixed rewriting (for rule-based languages), with functions (for functional languages), structures (for logic-like languages) and safe imperative structures, all 'glued together' by a pattern-matching algorithm that takes into account the imperative features. To our knowledge, no similar study has appeared in the literature.

We have presented a clean and compact formalisation of iRho in the proof assistant Coq. The Subject-reduction theorem, which is particularly tricky on paper, was proved in Coq with relatively little effort. The full proof development amounts approximately to 43Kbyte and the size of the .vo file is approximately 1Mbyte, working with CoqV7.2.

During the development we often had the feeling that the mathematical design was driven by both the machine-assisted certification and the software implementation, and that the feedback between these three phases (which are usually considered distinct) was crucial for making both safe software and safe theory.

We have experimented with a 'pattern' (in the sense of 'The Gang of Four' (Gamma et al. 1994)) called DIMPRO (Design-IMplement-PROve) to design safe software that respects its mathematical and functional specifications in toto. This pattern will be familiar to anyone who has knowledge of proof assistants.

Essentially, we started from a clean and elegant mathematical design, we continued with an implementation of a prototype satisfying the design, and, finally, we completed it

\footnotetext{
$\dagger$ 'A pattern is the abstraction from a concrete form which keeps recurring in specific non-arbitrary contexts...' (Riehle and Züllighoven 1996).
} 
with a mechanical certification of the mathematical properties of the design by looking for the simplest 'adequacy' property of the related software implementation. These three phases are strictly coupled and, very often, a particular choice in one phase induced a corresponding choice in another phase, which very often forced backtracking.

Refinement of this process was done by iterating cycles until all the required global properties were achieved (the process is reminiscent of a fixed-point computation, or a B-refinement (Abrial 1996)). All three phases have the same status, and each influences the other.

The lesson learned with iRho was that the hand of the math's designer must be in strict contact with the hand of the software's designer, which, in turn, must be in strict contact with the hand of the proof's certifier. Our software interpreter has been a good test of the 'methodology'. More generally, this methodology could be applied in the setting of raising quality software to the highest levels of the Common Criteria, CC (Common Criteria Consortium 2005) (from EAL5 to EAL7), or level five of the Capability Maturity Model, CMM. We have included in our schedule of future work the task of 'formalising' our novel DIMPRO pattern in the folklore of 'design patterns', and hope that it will be useful to the community developing safe software for crucial applications.

\section{Related work}

Some implementations of the untyped Rewriting Calculus (uRho) can be found in the literature. Among them we would like to mention:

- RhoStratego (Stratego Team 2003) is an implementation of an early version of the uRho (Cirstea and Kirchner 2001), written in the strategic language Stratego (Stratego Team 2005). The implementation tests strategic programming with higherorder functional programming.

- Rogue (Stump and Schürmann 2005) is another implementation of a dialect of the uRho (Cirstea and Kirchner 2001): this implementation is very interesting since some imperative features are added to the language, for example, reading and writing 'attributes' of expressions and a fixed strategy. Rogue has an interesting application, namely, it is the implementation language for building a new Validity Checker based on the CVC (Stump et al. 2002) infrastructure.

- JRho (Faure and Moreau 2002) is a Java prototype of uRho (Cirstea and Kirchner 2001) using the TOM pattern-matching compiler (Tom team 2003).

\section{Future directions}

The iRho calculus is suitable for extension with more powerful pattern-matching algorithms, and more sophisticated type systems capturing all modern object-oriented features, including both class-based and prototype-based ones. Among possible developments, we identify the following:

- To add an exception handling mechanism along the lines of Cirstea et al. (2002) - this would lead us to modify both the static and dynamic semantics. 
- To add a subtyping relation - this would allow one to type check considerably more programs in iRho by enhancing the type system with bounded polymorphism and object-types, together with the design of a type inference algorithm.

- To enhance the calculus with garbage collection - currently, new locations created during reduction remain in the store forever; extending the calculus with suitable modern exception mechanisms would be also worth studying.

- To analyse, perhaps using abstract interpretation or static analysis techniques, the possibility of statically catching some pattern-matching failures.

- To enhance our matching algorithm with residuation and narrowing in the style of the functional-logic programming language Curry by Michael Hanus (Hanus 1997).

- To add some ad hoc XML primitives to iRho.

- To enhance our proof development in order to reach software extraction using Coq this would be particularly appealing as it would eliminate one cycle in our DIMPRO pattern.

- To apply DIMPRO to the design of a simple compiler from iRho toward an abstract machine, such as JVM, or .NET, or to a variant of Landin's machine (Boudol and Zimmer 2002).

\section{Acknowledgments}

The authors would like to thank all the members of the Protheo Team in Nancy for their invaluable comments and interactions on the subject of the Rewriting Calculus. The authors also warmly thank Barry Jay for the fruitful $\mathrm{X}$-discussions, with $\mathrm{X} \in$ $\{@$, LondonPub, WSRewriting\}.

Liquori visited the University of Sussex, Brighton, and he would like to thank his hosts Matthew Hennessy and Vladimiro Sassone, and the whole Department of Informatics there for the ideal working conditions they provided.

Finally, the authors are very grateful to Matt Wall, Germain Faure, Daniel Dougherty and Steffen van Bakel for their careful reading of the paper, and to all the anonymous referees for their extremely useful comments and suggestions.

This work is supported by the French grant CNRS ACI Modulogic and by the AEOLUS FET Global Computing Proactive, Algorithmic Principles for Building Efficient Overlay Computers.

\section{References}

Abrial, J. R. (1996) The B-Book: Assigning Programs to Meanings, Cambridge University Press. Asf+Sdf Team (2005) The Asf+Sdf Meta-Environment Home Page. http://www.cwi.nl/htbin/ sen1/twiki/bin/view/SEN1/MetaEnvironment.

Barthe, G., Cirstea, H., Kirchner, C. and Liquori, L. (2003) Pure Pattern Type Systems. In: Proc. of POPL'03, The ACM Press.

Boudol, G. and Zimmer, P. (2002) Recursion in the Call-by-Value Lambda-Calculus. In: Proc. of FICS. Note Series NS-02-2. BRICS.

Cirstea, H. and Kirchner, C. (2001) The Rewriting Calculus - Parts I and II. Logic Journal of the Interest Group in Pure and Applied Logics 9 (3) 427-498. 
Cirstea, H., Kirchner, C. and Liquori, L. (2001a) Matching Power. In: Proc. of RTA. Springer-Verlag Lecture Notes in Computer Science 2051 77-92.

Cirstea, H., Kirchner, C. and Liquori, L. (2001b) The Rho Cube. In: Proc. of FOSSACS. SpringerVerlag Lecture Notes in Computer Science 2030 166-180.

Cirstea, H., Kirchner, C. and Liquori, L. (2002) Rewriting Calculus with(out) Types. In: Proc. of WRLA. Electronic Notes in Theoretical Computer Science.

Cirstea, H., Liquori, L. and Wack, B. (2004) Rho-calculus with Fixpoint: First-order system. In: Proc. of TYPES, Springer-Verlag.

Common Criteria Consortium (2005) The Common Criteria Home Page. http://www . commoncriteria.org.

Cousineau, G., Curien, P.-L. and Mauny, M. (1987) The Categorical Abstract Machine. Science of Computer Programming 8 (2) 173-202.

Cristal Team (2003) Concert: Compilateurs Certifiés. ARC INRIA 2003-2004. http://www-sop. inria.fr/lemme/concert.

Cristal Team (2005) OCaml Home page. http://www.ocaml.org/.

Faure, G. and Moreau, P. (2002) Jrho: a Java Implementation of the Rho Calculus. http://elan.loria.fr/Soft/jrho-0.1.tar.gz.

Felleisen, M. and Friedman, D. P. (1989) A Syntactic Theory of Sequential State. Theoretical Computer Science 69 (3) 243-287.

Frisch, A. (2005) The Cduce Home Page. http://www.cduce.org.

Gamma, E., Helm, R., Johnson, R. and Vlissides, J. M. (1994) Design Patterns Elements of Reusable Object-Oriented Software, Addison-Wesley.

Gimenez, E. (1998) Structural Recursive Definitions in Type Theory. In: Proc. of ICALP 397-408.

Goguen, J. (2005) The OBJ Family Home Page. http://www.cs.ucsd.edu/users/goguen/sys/ obj.html.

Hanus, M. (1997) A Unified Computation Model for Functional and Logic Programming. In: Proc. of POPL, The ACM Press 80-93.

Hirschowitz, T., Leroy, X. and Wells, J. B. (2003) Compilation of Extended Recursion in Call-byValue Functional Languages. In: In Proc. of PPDP, The ACM Press.

Huet, G. (1976) Résolution d'equations dans les langages d'ordre 1,2, ., ,, Ph.D. thesis, Université de Paris 7 (France).

Kahn, G. (1987) Natural Semantics. In: Proc. of STACS. Springer-Verlag Lecture Notes in Computer Science 247 22-39.

Kelsey, R., Clinger, W. and Rees, J. (1998) Revised ${ }^{5}$ Report on the Algorithmic Language Scheme. Higher-Order and Symbolic Computation 11 (1). (Also in ACM SIGPLAN Notices (1998) 33 (9).)

Klop, J. (1980) Combinatory Reduction Systems, Ph.D. Thesis, CWI Amsterdam. (Mathematical Centre Tracts, CWI, Amsterdam 127.)

Kowalski, R. (1979) Logic for Problem Solving, Artificial Intelligence Series, North Holland.

Landin, P. J. (1964) The Mechanical Evaluation of Expression. The Computer Journal 6 308-320.

Lee, K., LaMarca, A. and Chambers, C. (2003) HydroJ: Object-Oriented Pattern Matching for Evolvable Distributed Systems. In: Proc. of OOPSLA, The ACM Press.

Leroy, X. (2005) Formal certification of a compiler back-end, or: programming a compiler with a proof assistant. (Submitted.)

Liquori, L. and Serpette, B. (2005) Web Appendix to the current paper. http://www-sop.inria.fr/ mascotte/Luigi.Liquori/iRho.

Logical Team (2005) The Coq Home Page. http://coq.inria.fr.

Martin-Löf, P. (1984) Intuitionistic Type Theory, Studies in Proof Theory 1, Bibliopolis. 
Mason, I. A. and Talcott, C. L. (1992) References, Local Variables and Operational Reasoning. In: In Proc. of LICS 66-77.

Maude Team (2005) The Maude Home Page. http://maude.cs.uiuc.edu/.

Mendler, N. P. (1987) Inductive Definition in Type Theory, Ph.D. thesis, Cornell University.

Mendler, N. P., Panangaden, P. and Constable, R. L. (1986) Infinite Objects in Type Theory. In: Proc. of LICS 249-255.

Microsoft (2005) The C\# Home Page. http://msdn.microsoft.com/vcsharp/.

Milner, R. (1986-87) CS 3 Language Semantics. Course notes, LFCS, University of Edinburgh.

Milner, R., Tofte, M., Harper, R. and MacQueen, D. et al. (1997) The Definition of Standard ML (Revised), The MIT Press.

Morrisett, G., Felleisen, M. and Harper, R. (1995) Abstract Models of Memory Management. In: Proc. of FPCA, The ACM Press 66-77.

Peyton-Jones, S. (2003) Haskell 98 Language and Libraries, Cambridge University Press. (Also as a special issue in The Journal of Functional Programming (2003) 13 (1).

Plotkin, G. D. (1981) A Structured Approach to Operational Semantics. Technical Report DAIMI FN-19, Aarhus University.

Plotkin, G. D. (2004) The Origins of Structural Operational Semantics. J. Log. Algebr. Program. 60-61 3-15.

Protheo Team (2005) The Elan Home Page. http://elan.loria.fr.

Riehle, D. and Züllighoven, H. (1996) Understanding and Using Patterns in Software Development. In: Theory and Practice of Object Systems 2 (1) 3-13.

Serrano, M. (2005) The Scheme Bigloo Home Page. http://www.sop.inria.fr/mimosa/fp/ bigloo/.

Stratego Team (2003) The Rho Stratego Home Page.

http://www . stratego-language.org/twiki/bin/view/Stratego/RhoStratego.

Stratego Team (2005) The Stratego Home Page. http://www.stratego-language.org.

Stump, A., Barrett, C.W. and Dill, D. L. (2002) CVC: A Cooperating Validity Checker. In: 14th International Conference on Computer-Aided Verification.

Stump, A. and Schürmann, C. (2005) The Rogue Home Page. http://www.cse.wustl.edu/stump/ rogue.html.

Sun (2005) Java Technology. http://java. sun.com/.

Tofte, M. (1987) Operational Semantics and Polymorphic Type Inference, Ph.D. thesis, LFCS, University of Edinburgh.

Tom team (2003) The Tom Home Page. http://tom.loria.fr/.

Van Deursen, A., Heering, J. and Klint, P. (1996) Language Prototyping, World Scientific.

van Oostrom, V. (1990) Lambda Calculus with Patterns. Technical Report IR-228, Faculteit der Wiskunde en Informatica, Vrije Universiteit Amsterdam.

Xduce Team (2005) The Xduce Home Page. http://xduce.sourceforge.net. 